\title{
Fixed-grid numerical modeling of melting and solidification using variable thermo-physical properties - Application to the melting of n-Octadecane inside a spherical capsule
}

\author{
P. A. Galione ${ }^{\mathrm{a}, \mathrm{c}}$, O. Lehmkuhl ${ }^{\mathrm{a}, \mathrm{b}}$, J. Rigola ${ }^{\mathrm{a}}$, A. Oliva $\mathrm{a}^{\mathrm{a}, *}$ \\ ${ }^{a}$ Heat and Mass Transfer Technological Center (CTTC), Universitat Politècnica de Catalunya - BarcelonaTech, \\ ETSEIAT, Colom 11, 08222, Terrassa (Barcelona), Spain \\ ${ }^{b}$ Termo Fluids S.L., Av. Jacquard 977 1-E, 08222, Terrassa (Barcelona), Spain \\ ${ }^{c}$ Instituto de Ingeniería Mecánica y Producción Industrial (IIMPI), Universidad de la República (UdelaR), Julio \\ Herrera y Reissig 565, 11300, Montevideo, Uruguay
}

\begin{abstract}
A fixed-grid enthalpy model for unstructured meshes and explicit time integration schemes (P. A. Galione, O. Lehmkuhl, J. Rigola and A. Oliva, 2014 [1]) is here extended for taking into account the change in density and other thermo-physical properties with the temperature and phase. Thermal expansion and contraction associated to the phase change are taken into account in the conservation equations, and different strategies for the numerical treatment of the energy equation are discussed in detail. Further modifications to the original model are also presented.

The proposed model is used for simulating a case of melting of n-Octadecane inside a spherical capsule. Two and three-dimensional simulations are performed using constant and variable properties. The effect of adopting two different numerical schemes for the convective term of the energy equation is evaluated. A comprehensive examination of the thermo-physical properties is performed, and the different values and correlations used are here presented and criticised. Differences in the flow patterns are encountered between two and three-dimensional simulations. The effects of considering constant or variable properties are discussed. Two different thermal boundary conditions are tested and the results are compared against experimental data obtained from the literature.
\end{abstract}

Keywords: Melting and solidification, Phase change materials, Fixed-grid enthalpy models, n-Octadecane, Variable thermo-physical properties

\footnotetext{
${ }^{*}$ Corresponding author

Email addresses: pedrog@cttc.upc.edu, pgalione@fing.edu.uy (P. A. Galione), cttc@cttc.upc.edu (A. Oliva)
} 


\section{NOMENCLATURE}

A surface area

$C_{p} \quad$ specific heat at constant pressure

$\overrightarrow{d_{P F}} \quad$ distance vector, going from present node centroid to neighbour node centroid

$\overrightarrow{d_{P s f}} \quad$ distance vector, going from present node centroid to face centroid

$f \quad$ mass liquid fraction

$g \quad$ gravity acceleration

$h \quad$ specific enthalpy

$\hat{j} \quad$ unitary vector in the vertical direction

L $\quad$ specific latent enthalpy

$m, \dot{m} \quad$ mass and mass flux

$\hat{n} \quad$ surface normal vector

$p \quad$ pressure

$p_{d} \quad$ hydrodynamic part of the pressure $\left(p_{d}=p+\rho g y\right.$, where $y$ is the vertical coordenate)

$R \quad$ sum of convective, diffusive and buoyancy terms

$S \quad$ momentum source term coefficient introduced by the porosity method

$t \quad$ time

$T \quad$ temperature

$u, \vec{u}, \vec{u}^{p} \quad$ velocity magnitude, vector and predictor velocity vector

V volume

$\alpha_{e q} \quad$ equivalent heat transfer coefficient between the water bath and the internal suface of the capsule

$\beta \quad$ volumetric expansion coefficient

$\epsilon \quad$ volumetric liquid fraction (porosity)

$\lambda \quad$ thermal conductivity

$\mu \quad$ dynamic viscosity

$\rho \quad$ density

$\phi \quad$ non-dimensional temperature

Superscripts and subscripts: 


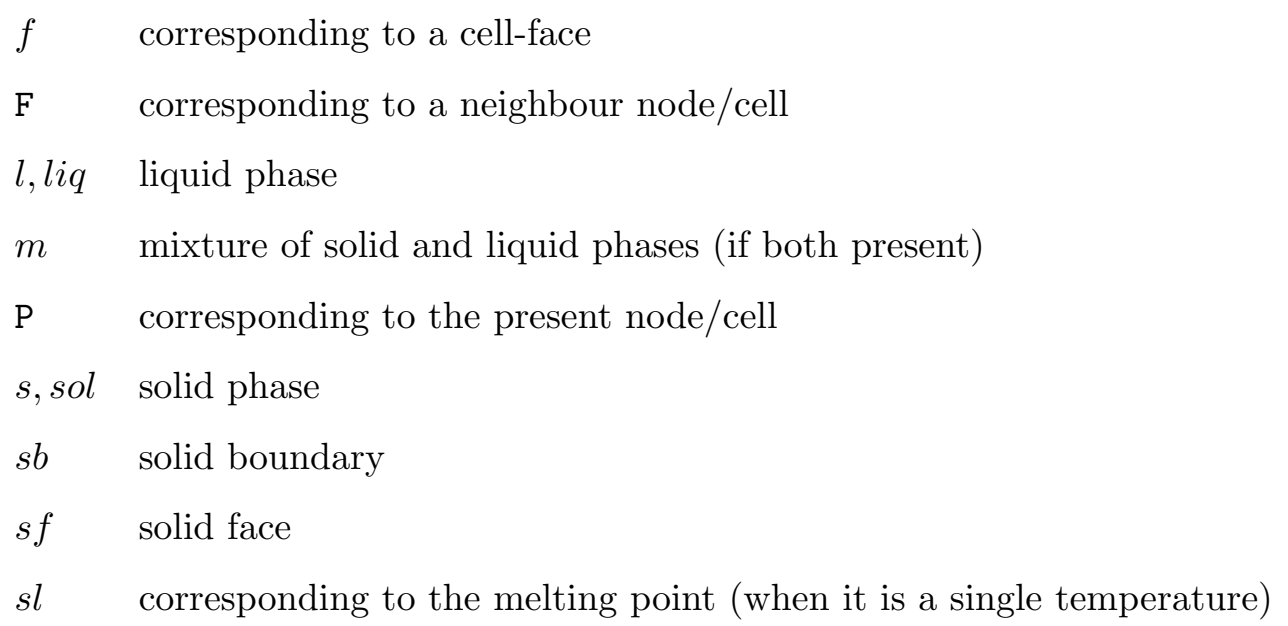

\section{Introduction}

Materials which undergo a phase transition at a temperature of interest for some application, are usually called phase change materials (PCMs). Due to the lower density changes involved, solidliquid phase change is usually preferred over liquid-vapor. This change of phase can be exploited, for example, to store thermal energy. Taking advantage of both latent and sensible energy capacities of the materials result in devices with higher energy densities, and therefore, allow the reduction of both the storage weight and volume. One application of PCMs where extensive research is being carried out nowadays, is the thermal energy storage for for concentrated solar power stations (CSP), see for instance $[2,3,4]$.

A common feature of many of the PCMs usually considered - such as paraffins, hydrated salts and salt mixtures - is their low thermal conductivity. For this reason, different strategies for increasing the heat transfer rate of devices using PCMs are considered $[4,5]$. One of the most studied thermal enhancement options is the containment of the PCM in capsules, in order to obtain a high surface-tovolume ratio. Studies of storage systems consisting of packed beds of encapsulated PCM are abundant in the literature, e.g. $[6,7,8]$.

It has been seen that natural convection effects in the melting of PCMs enclosed in different kind of cavities, play a significant role in the evolution of the melting rate and shape of the solid-liquid interface (see e.g. $[9,10]$ ). Besides the dynamics and heat transfer phenomena involved within the fluid phase, simulations need to address the heat transfer within the solid phase and an accurate representation of the moving boundary between phases. Different modeling strategies have been adopted for the numerical simulation of this kind of moving boundary problems, such as adapting grid (e.g. [11]), fixed-grid enthalpy (e.g. [12]) and Eulerian-Lagrangian (e.g. [13]) models. Within these, fixed-grid enthalpy models have been used more extensively, due to their relative mathematical 
and computational simplicity and their possibility to address the "mushy zones" produced in materials whose phase-change occurs in a temperature range. Generally, fixed-grid models have been used along with implicit time integration schemes (see e.g. [12, 14]).

Several works have studied the heat transfer in PCMs contained in spherical capsules. Assis et al. [15] performed a numerical and experimental study of the unconstrained melting of a phase change material inside a spherical capsule. Their numerical simulations were performed using the comercial CFD code ANSYS FLUENT 6.0 with an enthalpy-porosity model based on the works of Voller et al. [12], using variable thermo-physical properties and simulating the interface between air and PCM with a VOF technique. However, the numerical treatment of the change of density between solid and liquid phases is not clearly stated in their paper. Axi-symmetry was assumed in their simulations. Tan et al. [16] presented an experimental and numerical study of the melting of n-Octadecane contained in a spherical capsule. A hollow tube was placed in the axis of the sphere in order to hold the thermocouples and the solid phase from sinking to the bottom due to gravitational force. A two-dimensional (2D) model was used for the numerical simulations, assuming axi-symmetry with respect to the vertical axis. Qualitative agreement with experimental results was achieved, but a higher melting rate was reported for the numerical simulations. Discrepancies were believed to be caused due to not taking into account accurately the convection and thermal stratification of the thermal bath in which the capsule was immersed. Zhao et al. [17] performed transient 2D analysis of heat transfer in encapsulated PCMs, using both front-tracking and enthalpy-porosity methods. They validated their simulations with the enthalpy-porosity model using the constrained n-Octadecane melting case of Tan et al. [16], adopting constant thermo-physical properties. Results obtained showed a good agreement with the numerical results of Tan et al. [16], i.e. qualitative agreement with experimental results but a faster melting rate. Elmozughi et al. [18] conducted numerical simulations of melting and solidification in cylindrical and spherical capsules using the enthalpy-porosity method implemented in ANSYS FLUENT code. They used different properties for the liquid and solid phases of the PCM, but constant within each phase, and adopted a Boussinesq approximation in order to account for density differences in the liquid phase. However, the numerical treatment of the density differences between liquid and solid phases is not indicated in detail. Two and three-dimensional simulations of different cases were performed, although no particular 3D flow patterns were reported and axi-symmetry was observed.

No 3D simulations have been found in the literature for the case presented by [16], nor any 2D accounting for the variations of the properties with the temperature. Furthermore, no clarification about the numerical treatment of the flow produced by the expansion or contraction in the solid-liquid interface (if different from that with constant density) has been found in the previously mentioned 
works.

The present is a continuation of a previous work [1], which dealt with fixed-grid solid-liquid phasechange modeling using explicit time schemes, specially suited for its combination with turbulence models for simulation of the fluid motion. That model was tested using different benchmark cases found in the literature. Here, several upgrades are introduced into the formulation presented in [1].

First, the way the pressure is solved in the solid-liquid interface is modified by adopting a simpler strategy more convenient for situations of variable density. Furthermore, the model is extended to take into account variations of the thermo-physical properties with the temperature, including the density differences between solid and liquid phases and also within the liquid phase. The resulting modifications on the treatment of the three conservation equations (mass, momentum and energy) are discussed in detail. The strategies for calculating the temperature and liquid fraction from the enthalpy are presented for both materials which change of phase at a fixed temperature and in a temperature range. Furthermore, a variation in the form of the source term is proposed and used in the simulations presented.

The improved numerical model is applied for simulating the melting of a phase-change material (PCM) contained in a spherical capsule, based on the work of Tan et al. [16]. Here, simulations of the case of Tan et al. [16] are presented with the proposed formulation, using two and three-dimensional (3D) unstructured meshes and taking into account the variation of properties with temperature and phase. Unlike several previous works $[12,14,15,16,17,18]$ a clear distinction between liquid and solid properties is performed in the equations, and more specifically, only the liquid phase is considered in the convective terms due to being the only transporting phase, as suggested in [1]. Furthermore, the effect of adopting two different numerical schemes for discretizing the convective term of the energy equation is analyzed.

In order to perform accurate numerical simulations of the melting of n-Octadecane, a comprehensive examination of its physical properties has been performed. Correlations used are presented along with comments about their validity. Simulations have been run using with both constant and variable thermo-physical properties and their results compared. Results showing the development of the interface, as well as the evolution of the temperature at some selected positions, are presented and compared against the experimental results obtained from [16]. Results show that the 3D treatment allows to reproduce flow patterns that are not simulated with the $2 \mathrm{D}$ models and are in better agreement with experimental results. 


\section{Mathematical modeling}

The equations presented in [1] for modelling solid-liquid phase change CFD are here used and extended to variable physical properties. Specifically, the density is allowed to vary between solid and liquid phases and within the liquid phase. This introduces an important modification to the model, since the traditional incompressibility condition $(\nabla \cdot \vec{u}=0)$ is not valid anymore.

Resulting governing equations (mass, momentum and energy balances) are:

$$
\begin{gathered}
\frac{\partial \rho_{m}}{\partial t}+\nabla \cdot\left(\rho_{l} \vec{u}\right)=0 \\
\frac{\partial \rho_{l} \vec{u}}{\partial t}+\nabla \cdot\left(\rho_{l} \vec{u} \frac{\vec{u}^{*}}{\epsilon}\right)=-\nabla p+\nabla \cdot\left(\mu_{l} \nabla \vec{u}\right)+\rho_{l} \epsilon \vec{g}-S \vec{u} \\
\frac{\partial \rho_{m} h_{m}}{\partial t}+\nabla \cdot\left(\rho_{l} h_{l} \vec{u}\right)=\nabla \cdot\left(\lambda_{m} \nabla T\right)
\end{gathered}
$$

where:

$$
\begin{gathered}
\rho_{m}=\epsilon \rho_{l}+(1-\epsilon) \rho_{s} \\
\rho_{m} h_{m}=\epsilon \rho_{l} h_{l}+(1-\epsilon) \rho_{s} h_{s}
\end{gathered}
$$

Sub-indexes $l$ and $s$ indicate properties of the liquid and solid phases, respectivley; while $m$ indicates a property of the mixture of solid and liquid phases (including the cases of pure liquid and solid). $\epsilon$ is the volumetric liquid fraction, which can take values between 0 (solid) and 1 (liquid). Velocity $\vec{u}$ corresponds to the fluid velocity or to the seepage (or Darcy) velocity [19] in the presence of a mixture of solid and liquid phases. Enthalpy $h$ include both liquid and sensible components.

Thermal conductivity $\lambda_{m}$ is an effective conductivity, which can be either the conductivity of the pure phase - in the mesh cells where there is only one phase present- or a combination of the conductivities of both phases - in the cells where there is a mixture of solid and liquid (interface). If the interface zone is a "mushy" zone, this value could be calculated using some of the correlations used for the heat transfer in porous media [19, 20].

Equation (2) corresponds to the momentum equation for flow through a porous medium [21], where the momentum source term coefficient $S$ has been simplified, only accounting for the Darcian part of the drag force induced by the presence of the solid phase, as in [22]. This equation is only useful in the liquid-containing domain.

It has been assumed that the solid phase has no motion, and therefore, the transport of properties is carried out by the liquid phase only. This is the reason for the advective terms only to include the properties of the liquid phase, as mentioned in [1]. 
If using constant physical properties, Boussinesq approximation is performed in order to account for the thermal expansion in the fluid.

\subsection{Enthalpy - temperature relations}

The energy conservation equation (3) is expressed in terms of enthalpy in its transient and convective terms. Therefore, relations between enthalpy and temperature have to be used explicitly in order to be able to determine the thermodynamic state of the material. Since the aim is to be able to take into account the variation of the thermo-physical properties with the temperature, the variation of the specific heat $\left(C_{p}\right)$ and density are considered.

Next, this task is discussed in some detail for cases of phase change occurring at a fixed temperature (for pure substances) or in a temperature range (for mixtures of substances).

\subsubsection{Fixed phase-change temperature}

In pure substances, the melting point is a fixed value. Therefore, the change in enthalpy in the phase change is only due to changes in the amount of substance that changes from liquid to solid, or viceversa. Hence, there is no function relating $h$ and $T$ but there is a function relating $h$ and $f$ (mass liquid fraction) instead.

For the liquid and solid phases, relations between $h$ and $T$ exist, being the usual relations for sensible enthalpy changes:

$$
\begin{array}{rlr}
h_{s}-h_{\text {ref }}=\underbrace{\int_{T_{r e f}}^{T} C_{p, s} d T}_{\text {sensible }} & \left(T<T_{s l}\right) \\
h_{l}-h_{\text {ref }}=\underbrace{\int_{T_{s l}}^{T} C_{p, l} d T}_{\text {sensible liquid }}+\underbrace{\int_{T_{r e f}}^{T_{s l}} C_{p, s} d T}_{\text {sensible solid until m.p. }}+\underbrace{L}_{\text {latent }} & \left(T>T_{s l}\right)
\end{array}
$$

For the interface $\left(T=T_{s l}\right)$ the enthalpy can be calculated from:

$$
h_{m}-h_{r e f}=\underbrace{\int_{T_{r e f}}^{T_{s l}} C_{p, s} d T}_{\text {sensible solid until m.p. }}+\underbrace{f L}_{\text {latent }}
$$

with:

$$
f=\frac{m_{l}}{m_{l}+m_{s}}=\frac{h_{m}-h_{s_{@} T_{s l}}}{h_{l_{@ T_{s l}}-h_{s_{@} T_{s l}}}}
$$

131 The "thermodynamic state" of the mixture is determined by the mass liquid fraction $f$, which is calculated from the enthalpy of the mixture $h_{m}$. 
Depending on the values of $C_{p}$ for both phases, the inversion of the relation $h-T$ can be more or less difficult. The easiest case is that with constant values of $C_{p}$ for both phases, resulting in:

$$
\begin{array}{ll}
T=T_{r e f}+\frac{h_{s}-h_{r e f}}{C_{p, s}} & \left(T<T_{s l}\right) \\
T=T_{s l}+\frac{h_{l}-\left[h_{r e f}+C_{p, s}\left(T_{s l}-T_{r e f}\right)+L\right]}{C_{p, l}} & \left(T>T_{s l}\right)
\end{array}
$$

However, more complicated expressions for $C_{p}(T)$ are usually found, which can make the work of inverting the $h-T$ relations very hard. A useful way of easing this task is to piecewise-linearize these expressions in some temperature ranges. Since the values of $C_{p}$ for each phase generally do not change significantly in the working range of temperatures, a single interval of linearization might be sufficient (this is the approach that has been adopted in this work for the n-Octadecane). If this were the case, the values of $C_{p}$ would be expressed as:

$$
\begin{array}{rlrl}
C_{p, s} & =C_{p, s_{@} T_{\text {min }}}+\frac{C_{p, s @ T_{s l}}-C_{p, s @ T_{\min }}\left(T-T_{\text {min }}\right)}{T_{s l}-T_{\text {min }}} & & \left(T_{\text {min }}<T<T_{s l}\right) \\
C_{p, l}=C_{p, l @ T_{s l}}+\frac{C_{p, l @ T_{\text {max }}}-C_{p, l_{@} T_{s l}}}{T_{\text {max }}-T_{s l}}\left(T-T_{s l}\right) & \left(T_{s l}<T<T_{\text {max }}\right)
\end{array}
$$

or, expressed in a generic linear form:

$$
C_{p}=C_{p 0}+C_{p 1} T
$$

resulting in the following generic expression for $h$ depending on $T$ :

$$
h-h_{0}=C_{p 0}\left(T-T_{0}\right)+0.5 C_{p 1}\left(T^{2}-T_{0}^{2}\right)
$$

where the 0 represents either the melting point $s l$ (for the liquid range) or the reference state $r e f$ (for the solid range). From here on, $T$ is calculated as:

$$
T=-C_{p 0} / C_{p 1}+\sqrt{2\left(\frac{h-h_{0}}{C_{p 1}}\right)+\left(\frac{C_{p 0}}{C_{p 1}}+T_{0}\right)^{2}}
$$

In the interface, instead of using the mass liquid fraction $f$ it is useful to use the volumetric liquid fraction (or porosity) $\epsilon$, since it is a variable that is explicitly used in the momentum equation. Hence, using Eq. (5), the following equation for $\epsilon$ can be obtained:

$$
\epsilon=\frac{\rho_{m} h_{m}-\rho_{s} h_{s}}{\rho_{l} h_{l}-\rho_{s} h_{s}}
$$


Furthermore, using Eq. (4), the following expression — which does not depend on $\rho_{m}$ - is obtained:

$$
\epsilon=\frac{\rho_{s}\left(h_{m}-h_{s}\right)}{\rho_{l} h_{l}-\rho_{s} h_{s}+\left(\rho_{s}-\rho_{l}\right) h_{m}}
$$

Since $\rho_{l}, \rho_{s}, h_{l}$ and $h_{s}$ are all fixed and known values at $T=T_{s l}$, the porosity can be directly calculated from Eq. (7) having previously calculated $h_{m}$ from the energy equation. It should be noted that Eq. (7) is not linear with $h_{m}$ in the general case, since $h_{m}$ is also present in the denominator. In the particular case of having equal solid and liquid densities, the expression results to be linear.

It should be noted that with equation (7), the porosity is calculated directly from the enthalpy $\left(h_{m}\right)$ and not from the temperature, as is usually the case when an implicit time integration is used (e.g. $[12,14,16])$, avoiding the need for a numerical maneuver such as the definition of an artificial range of temperatures (small) in which the material is assumed to melt, in order to have an expression of $\epsilon$ as a function of $T$.

\subsubsection{Phase-change in a temperature range}

The mixture of two or more substances do not usually change of phase at a sharp melting point, but they have a range of temperatures in which both phases coexist. In these cases, there exist a region in which both phases are present, called "mushy" zone, and the interface is not a surface anymore.

Being $T_{s}$ and $T_{l}$ and $h_{s_{@ T_{s}}}$ and $h_{l_{@ T_{l}}}$ the solidus and liquidus temperatures and enthalpies, respectively; the enthalpies of the liquid and solid phases, $h_{l}$ and $h_{s}$, could be defined as follows:

$$
\begin{array}{rlr}
h_{l}=h_{l_{@ T_{l}}}+\int_{T_{l}}^{T} C_{p, l}(T) d T & \left(T>T_{s}\right) \\
h_{s}=h_{s_{@} T_{s}}-\int_{T}^{T_{s}} C_{p, s}(T) d T & \left(T<T_{l}\right) \\
\rho_{l}=\mathrm{f}_{1}(T) & \left(T>T_{s}\right) \\
\rho_{m}=\epsilon \rho_{l}+(1-\epsilon) \rho_{s} & \left(T_{s}<T<T_{l}\right) \\
\epsilon=\frac{\rho_{m} h_{m}-\rho_{s} h_{s}}{\rho_{l} h_{l}-\rho_{s} h_{s}} & \left(T_{s}<T<T_{l}\right)
\end{array}
$$

where $\rho_{s}$ has been assumed to be constant over all the range $T<T_{l}, \rho_{l}$ is some function of the temperature $\left(f_{1}\right)$, and the last is Eq. (6). It should be noted that the equations of $h_{l}, h_{s}$ and $\rho_{l}$ have been assumed to be valid also in the whole phase change range; and differently from what happened with a fixed melting point, these properties are not constant in the phase change range.

The first three equations are valid for the pure phases and the interface, while the other two are only for the interface. These, added to the energy equation (3), are six equations for determining 
seven unknowns $\left(h_{l}, h_{s}, h_{m}, \rho_{l}, \rho_{m}, \epsilon, T\right)$. Therefore, an additional equation is necessary, which could be the relation between $f$ and $T$ (transformed to $\epsilon-T$ ) coming from the phase diagram of the mixture of substances.

Usually the $f-T$ relation is assumed as linear between the liquidus and solidus temperatures, but more accurate relations could be implemented, e.g. by using the lever rule in binary alloys.

To calculate all the properties, it is necessary to determine $T$ and $\epsilon$ from $h_{m}$, since $T$ is necessary for calculating $h_{l}, h_{s}, \rho_{l}$ and $\rho_{s}$. This will probably result in an iterative procedure, unless the extra equation is the relation between $h_{m}$ and $T$, which would allow to directly determine $T$ and from there on, to calculate directly the rest of the properties.

\section{Numerical implementation}

The formulation presented above has been implemented into the CFD computer package TermoFluids [23], which is designed to work with unstructured meshes. Finite volume, collocated discretization and explicit time schemes have been used, with the time step dynamically adapted by estimating upper bounds for the eigenvalues of convective and diffusive operators [24]. The code is parallelized and can be run both on single computers as well as in computing clusters.

Figure 1 shows some sketches of unstructured meshes, where the geometrical notation has been introduced. For more information about the way the different terms of the conservation equations have been discretized, please refer to $[1,25]$.

In order to better understand the modifications performed to the previous implementation (presented in [1]), a brief overview of the latter is presented in the first sub-section. Afterwards, the new treatment of mass and momentum equations, momentum source term coefficient, pressure in the interface zone and energy equations is described.

\subsection{Overview of the previous implementation}

For the non-dimensionalized problem, with constant thermo-physical properties, the time-discretization of governing equations (1)-(3), result in [1]:

$$
\begin{gathered}
\nabla \cdot \vec{u}^{n+1}=0 \\
\frac{\vec{u}^{n+1}-\vec{u}^{n}}{\Delta t}=R^{n+\alpha}(\vec{u})-\nabla p_{d}^{n+1}-S \vec{u}^{n+1} \\
\frac{h^{n+1}-h^{n}}{\Delta t}=R^{n+\alpha}(\phi)
\end{gathered}
$$


Here, $R^{n+\alpha}$ represents the sum of convective, diffusive and buoyancy terms integrated between time steps $n$ and $n+1$ using some time-integration scheme, $S$ is the non-dimensional source term coefficient introduced by the porous medium treatment and $\phi$ is the non-dimensional temperature. As explained in [1], the momentum source term has to be treated implicitly due to stability reasons.

Decoupling of $u^{n+1}$ and $p_{d}^{n+1}$ is carried out applying divergence, imposing mass conservation [Eq. (8)] and rearranging terms:

$$
\Delta t \nabla \cdot\left(\frac{\nabla p_{d}^{n+1}}{1+\Delta t S}\right)=\nabla \cdot u^{p}
$$

where:

$$
u^{p}=\left\{\frac{\vec{u}^{n}+\Delta t R^{n+\alpha}(\vec{u})}{1+\Delta t S}\right\}
$$

is a pseudo-predictor velocity, and thus:

$$
u^{n+1}=u^{p}-\Delta t\left(\frac{\nabla p_{d}^{n+1}}{1+\Delta t S}\right)
$$

from where the new velocity field $\left(u^{n+1}\right)$ is calculated. Equation (11) defines a linear system of equations of cell-node pressures, which needs to be solved using a linear solver.

The algorithm for the resolution of the equations is the following:

- Energy equation (10): new enthalpy, temperature and porosity fields are calculated.

- Pseudo-predictor velocities $u^{p}$ using previous velocity fields [Eq. (12)].

- Pressure equations (11): calculation of the system coefficients and resolution using a linear solver (e.g. CG).

- New velocity field using predictor velocity and new pressure field [Eq. (13)].

\subsection{Mass and momentum}

Numerical implementation of the mass balance is modified by adding the effect of density variations:

$$
\frac{\rho_{m}^{n+1}-\rho_{m}^{n}}{\Delta t}=-\nabla \cdot\left(\rho_{l}^{n+1} \vec{u}^{n+1}\right)
$$

Resulting in a modified version of the Poisson-like equations from those presented in [1]:

$$
\Delta t \nabla \cdot\left(\frac{\nabla p^{n+1}}{1+\Delta t S / \rho_{l}^{n+1}}\right)=\nabla \cdot\left(\frac{\rho_{l} u^{p}}{1+\Delta t S / \rho_{l}^{n+1}}\right)+\frac{\rho_{m}^{n+1}-\rho_{m}^{n}}{\Delta t}
$$

where, using Adams-Bashforth $2^{\text {nd }}$ order time integration scheme (for simplicity):

$$
\rho_{l} u^{p}=\rho_{l} \vec{u}^{n}+\Delta t\left[\frac{3}{2} R^{n}(\vec{u})-\frac{1}{2} R^{n-1}(\vec{u})\right]
$$




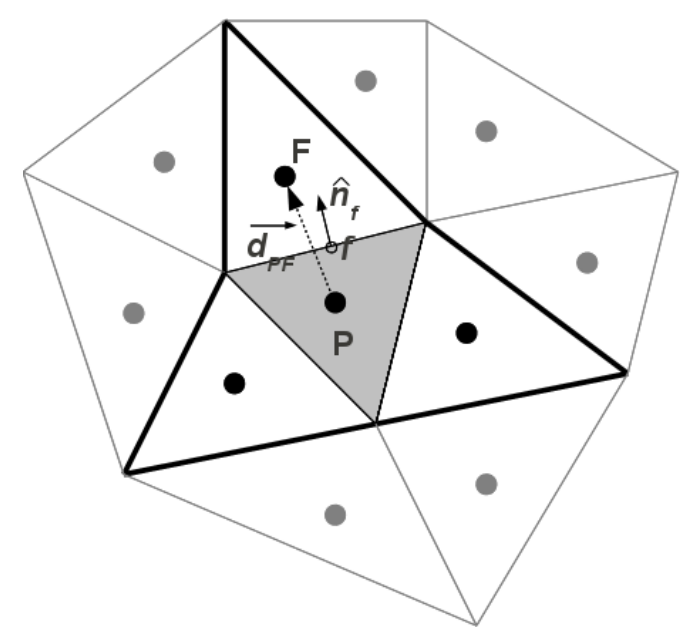

(a) Away from solid nodes

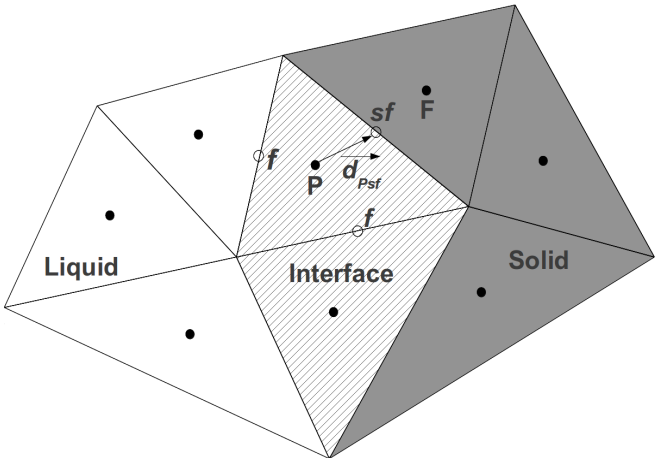

(b) Near solid nodes. Diferent phases are indicated by the area filling.

Figure 1: Notation used for the position of the nodes in an unstructured mesh. Letter P indicates the node being calculated, $\mathrm{F}$ a neighbouring node, $f$ the face shared by nodes $\mathrm{P}$ and $\mathrm{F}, \hat{n}_{f}$ the vector normal to face $f$ pointing outwards $\mathrm{P}$, and $\overrightarrow{d_{P F}}$ the vector going from $\mathrm{P}$ to $\mathrm{F}$. If node $\mathrm{F}$ contains solid, then the face connecting nodes $\mathrm{P}$ and $\mathrm{F}$ is denoted $s f$ and the distance from $\mathrm{P}$ to this face $\overrightarrow{d_{P s f}}$.

is a pseudo-predictor velocity, and thus:

$$
\rho_{l} u^{n+1}=\left(\frac{\rho_{l} u^{p}-\Delta t \nabla p^{n+1}}{1+\Delta t S / \rho_{l}^{n+1}}\right)
$$

from where the new velocity field $\left(u^{n+1}\right)$ is calculated. $R$ is the sum of convective, diffusive and buoyancy source terms.

The change in density is taken into account in the last term of the right hand side of Eq. (15). The new density $\left(\rho_{m}^{n+1}\right)$ is assumed to be known at this point, since the energy equation is resolved before than momentum, and therefore, the new temperature field and thermo-physical properties are known.

In the case of a fixed melting point, Eq. (4) can be used to calculate the change in density in the interface cells:

$$
\frac{\rho_{m}^{n+1}-\rho_{m}^{n}}{\Delta t}=\frac{\epsilon^{n+1}-\epsilon^{n}}{\Delta t}\left(\rho_{l}-\rho_{s}\right)
$$

which could be plugged into Eq. (15); e.g. when $\rho_{l}$ is considered constant and the Boussinesq approximation is used for taking into account the thermal expansion/contraction in the liquid phase.

It should be noted that $u^{p}$ in Eq. (16) does not include the effect of the source term coefficient $S$. This effect is included afterwards, when the velocity is corrected by the effect of the pressure gradient, in Eq. (17). This approach is different from that chosen in [1], where the effect of the momentum source term was included in the predictor velocity already. The modification presented here results 
in an interpolation of the face velocities more consistent with that used in the pressure terms in [1], which do not include the effect of the source term until the moment of performing the integration of the pressure gradient [first term of Eq. (15)].

Integrating Eq. (15) in space over a mesh cell and discretizing, it remains:

$$
\frac{\Delta t}{V_{\mathrm{P}}} \sum_{f} \underbrace{\left[\left(\frac{\nabla p^{n+1}}{1+\Delta t S / \rho_{l}^{n+1}}\right)_{f} \cdot \hat{n}_{f} A_{f}\right]}_{\text {modified pressure gradient flux }}-\frac{1}{V_{\mathrm{P}}} \sum_{f} \underbrace{\left\{\left[\frac{\left(\rho_{l} \vec{u}\right)^{p}}{1+\Delta t S / \rho_{l}^{n+1}}\right]_{f} \cdot \hat{n}_{f} A_{f}\right\}}_{\text {modified predictor velocity flux }}=\underbrace{\frac{\rho_{m, \mathrm{P}}^{n+1}-\rho_{m, \mathrm{P}}^{n}}{\Delta t}}_{\text {thermal expansion }}
$$

which, in short, stands for mass conservation:

$$
\frac{1}{V_{\mathrm{P}}} \sum_{f_{\mathrm{P}}} \dot{m}_{f}^{n+1}=\frac{\rho_{m, \mathrm{P}}^{n+1}-\rho_{m, \mathrm{P}}^{n}}{\Delta t}
$$

where the outgoing mass fluxes through faces are calculated as:

$$
\dot{m}_{f}^{n+1}=\frac{\dot{m}_{f}^{p}-\Delta t \nabla p^{n+1} \cdot \hat{n}_{f} A_{f}}{\left(1+\Delta t S / \rho_{l}\right)_{f}}
$$

The resulting system of pressure equations can be written as:

$$
a_{\mathrm{P}} \tilde{p}_{\mathrm{P}}^{n+1}+\sum_{f_{\mathrm{P}}} a_{\mathrm{F}_{f}} \tilde{p}_{\mathrm{F}_{f}}^{n+1}=b_{\mathrm{P}}
$$

where $\tilde{p}=\Delta t p$ and:

$$
\begin{aligned}
& a_{\mathrm{F}_{f}}=\frac{A_{f}}{\left(\overrightarrow{d_{P F}} \cdot \hat{n}\right)_{f}} \frac{\left(\theta_{F_{f}}+\theta_{P}\right)}{2} \\
& a_{\mathrm{P}}=\sum_{f_{\mathrm{P}}}-a_{\mathrm{F}_{f}} \\
& b_{\mathrm{P}}=\sum_{f_{\mathrm{P}}}\left[\dot{m}_{f}^{p} \frac{\left(\theta_{F_{f}}+\theta_{P}\right)}{2}\right]+V_{\mathrm{P}} \frac{\rho_{m, \mathrm{P}}^{n+1}-\rho_{m, \mathrm{P}}^{n}}{\Delta t} \\
& \theta_{i}=\frac{1}{1+\Delta t S_{i} / \rho_{l, i}^{n+1}} \quad\left(i=\mathrm{F}_{f}, \mathrm{P}\right)
\end{aligned}
$$

\subsubsection{Momentum source term coefficient (S)}

It can be observed [Eq. (17)] that the source-term coefficient $S$ produces a damping of the new velocity by a factor of

$$
\frac{1}{1+\Delta t S / \rho_{l}}
$$

In [1], different forms of the momentum source-term coefficient $(S)$ were studied in terms of the "smoothness" of the associated velocity damping produced. In particular, the approach proposed by Voller et al. [12]

$$
S=\frac{C(1-\epsilon)^{2}}{\epsilon^{3}+q}
$$




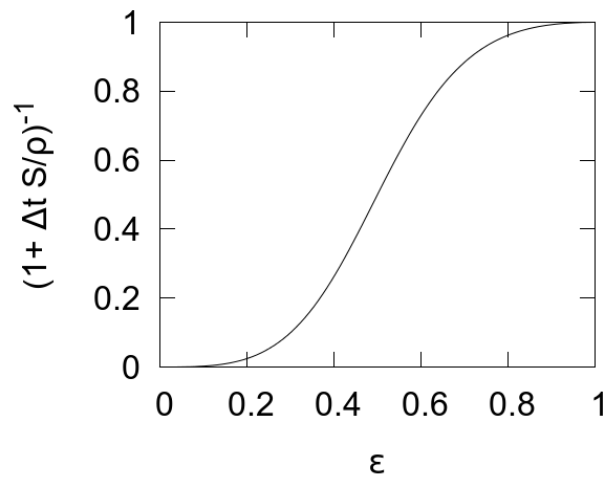

Figure 2: Damping factor vs. $\epsilon$, with the proposed approach

where $C$ is an arbitrary (big) constant and $q$ is a very small value included to avoid division by zero, resulted to be the best of those studied, in terms of smoothness.

However, analyzing equations (22) and (23), it can be seen that the damping effect depends on the values of $C, \rho_{l}$ and $\Delta t$. In the case of a porous medium, there are expressions to calculate the value of $C$ from the thermo-physical properties of the fluid [19]. In the case of a change of phase in a temperature range, where a mushy zone is formed, some of these expressions should be used. On the other hand, as stated in [14] and [1], in the case of a change of phase at a fixed temperature there is no mushy zone and the interface should be of infinitesimal width. Therefore, there is no physical meaning of the source term, and it is just intended to act as a velocity damper in the solid-containing cells.

With this in mind, a new strategy is followed for the choice of $S$. The idea is to retain the smoothness obtained with Eq. (23), with respect to the value of $\epsilon$, with independence of the values of $\Delta t$ and $\rho$. Hence, a damping of 0.5 is fixed for $\epsilon=0.5$, resulting in:

$$
S=\frac{\rho_{l}}{2 \Delta t}\left[\frac{(1-\epsilon)^{2}}{\epsilon^{3}+q}\right]
$$

and (disregarding $q$ ):

$$
\frac{1}{1+\Delta t S / \rho_{l}}=\frac{2 \epsilon^{3}}{2 \epsilon^{3}+(1-\epsilon)^{2}}
$$

The resulting curve of the damping factor vs. $\epsilon$ is shown in figure 2 .

\subsubsection{Solid boundary conditions}

Solid, no-slip, boundary conditions are treated in detail in this section; not only because they are extensively used in the study cases but also because they are related to the way the solid-liquid 
interface inside the domain have been treated in [1]. A modification to such strategy is the issue of the next section.

The velocity and mass flux in the solid boundaries are fixed to zero, the predictor velocity is not calculated since it is not needed (pressure of the boundary not included in the pressure system of equations, as in [1]):

$$
\vec{u}_{s b}=0 ; \quad \dot{m}_{s b}=0
$$

The pressure is needed for calculating the pressure gradient of the cell and is determined in order to give a pressure gradient such that the cell-centered velocity results parallel to the boundary:

$$
\vec{u}_{P} \cdot \hat{n}_{s b}=0
$$

thus, using Eq. (17):

$$
\begin{gathered}
\vec{\rho}_{l} u_{P} \cdot \hat{n}_{s b}=\left.\left(\frac{\rho_{l} \vec{u}^{p}-\Delta t \nabla p}{1+\Delta t S / \rho_{l}}\right)\right|_{P} \cdot \hat{n}_{s b}=0 \\
\Rightarrow\left(\rho_{l} \vec{u}^{p}-\Delta t \nabla p\right)_{P} \cdot \hat{n}_{s b}=0
\end{gathered}
$$

which, integrating and discretizing over the cell containing node $P$, gives:

$$
\begin{gathered}
\left.\frac{V \rho_{l} \vec{u}^{p}}{\Delta t}\right|_{P} \cdot \hat{n}_{s b}-\sum_{f_{P}} p_{f} \hat{n}_{f} A_{f} \cdot \hat{n}_{s b}=0 \Rightarrow \\
p_{s b} A_{s b}=\left.\frac{V \rho_{l} \vec{u}^{p}}{\Delta t}\right|_{P} \cdot \hat{n}_{s b}-\sum_{f \neq s b} p_{f} \hat{n}_{f} A_{f} \cdot \hat{n}_{s b}
\end{gathered}
$$

from where the pressure of the solid boundary $p_{s b}$ is calculated, after having determined the variables $\vec{u}^{p}$ and $p$ in the cells.

This condition on pressure in fact arises from a $2^{\text {nd }}$ order boundary condition for $\vec{u} \cdot \hat{n}_{s b}$, as is shown next. Due to mass conservation (neglecting the possible change in fluid density), the component of the velocity normal to the solid face should be zero:

$$
\nabla \cdot \vec{u}_{s b}=0 \Rightarrow \frac{\partial u_{t / s b}}{\partial x_{t_{s b}}}+\frac{\partial u_{t / s b}}{\partial x_{t_{s b}}}+\frac{\partial u_{n}}{\partial x_{n_{s b}}}=\left.0 \Rightarrow \frac{\partial u_{n}}{\partial x_{n}}\right|_{s b}=0
$$

where $\hat{t_{1 s b}}$ and $\hat{t_{2 s b}}$ are the two components tangent to the solid face, over which the velocity is zero in all the solid boundary. Therefore, the component of the cell-centered velocity normal to the solid boundary can be approximated as: 


$$
\begin{aligned}
\overrightarrow{u_{P}} \cdot \hat{n}_{s b} & =\overrightarrow{y_{s b}} \cdot \hat{n}_{s b}+\left.\frac{\partial u_{n}}{\partial x_{n}}\right|_{s b} ^{0} \Delta x_{n}+O\left(\Delta x_{n}^{2}\right) \\
& \Rightarrow \overrightarrow{u_{P}} \cdot \hat{n}_{s b}=0+O\left(\Delta x_{n}^{2}\right)
\end{aligned}
$$

from where it can be seen that Eq. (24) results in a second order approximation of $\overrightarrow{u_{P}} \cdot \hat{n}_{s b}$.

It should be pointed out that this boundary condition for pressure is applied due to being using a collocated mesh scheme, where the velocities at the center of the cells are not the ones directly involved in the mass conservation. The pressure system of equations involves the mass fluxes through the cell faces, and therefore, the cell-centered pressure values are determined in order to enforce mass conservation of those mass fluxes. For this task, the pressure of the solid boundaries is not used nor needed (as seen in [1]), which is equivalent to adopting the usual boundary condition for pressure in solid faces $\left(\frac{\partial p}{\partial n}=0\right)$ in the poisson-like system of equations.

If $\frac{\partial p}{\partial n}=0$ is used as the boundary condition for calculating the pressure in the solid faces, large normal velocities may result due to the effect of body forces (e.g. gravitational), which are not correctly counteracted by the resulting pressure gradient. This effect is mentioned in [26] and has been experienced by the authors.

However, the cell-centered velocities are calculated afterwards, and involve the calculation of the pressure gradient over the cell. This pressure gradient is the one using the value of the pressure in the solid boundary and is the one affected by the boundary condition imposed.

Furthermore, the boundary condition imposed by Eq. 25 ensures that the viscous stress normal to the solid boundary is zero as it should be [26], without needing to modify the diffusive term explicitly:

$$
\begin{aligned}
\nabla \cdot(\mu \nabla \vec{u}) & \simeq \sum_{f} \mu_{f} \nabla \vec{u} \cdot \hat{n}_{f}= \\
& \sum_{f \neq s b} \mu_{f} \nabla \vec{u} \cdot \hat{n}_{f}+\mu_{s b}\left(\left.\frac{\partial u_{n}}{\partial x_{n}}\right|_{s b} ^{0} \hat{n}_{s b}+\frac{\partial u_{t_{1}}}{\partial x_{n}} \hat{t}_{1 s b}+\frac{\partial u_{t_{2}}}{\partial x_{n}} \hat{t}_{2 s b}\right)
\end{aligned}
$$

\subsubsection{Pressure equation - cells close to the solid phase}

The pressure field effectively acts in the liquid domain, where it enforces mass conservation. In the solid domain it is not needed and it is required not to produce artificial "filtration" of flow through the interface into (or out from) the solid domain. This filtration effect could be produced due to the fact that between neighbouring liquid and solid cells, resulting from the solution of the system of equations, the pressure values will generally be different, and therefore, mass flows through the faces could be different from zero if calculated using Eq. (20). 
As in [1], the strategy is to decouple liquid (or liquid-containing) and solid domains by modifying the pressure system of equations. In [1], this decoupling was performed treating the solid-cells faces as solid boundaries. This internal boundary condition was applied to the faces of the solid cells in the same way as for the solid boundaries of the domain, enforcing the cell-centered velocities to be parallel to these internal solid boundaries (see section 3.2.2).

This strategy resulted in a fluid velocity field - near the solid phase - that changed direction following the shape of the solid cells, specially where totally liquid cells are in contact with totally solid ones. Since the interface is not exactly determined and its shape depends on the shape of the mesh cells, this "internal solid boundary" is usually highly irregular (this particularly true in the case of a 3D unstructured mesh of a spherical capsule), and therefore, to enforce the velocity field to follow this artificial boundaries does not necessarily result in an accurate representation of the flow.

Moreover, the implementation of such internal boundary condition is quite complex in terms of program coding, since different possibilities have to be taken into account regarding the number of solid neighbors of a liquid-containing cell; i.e. if the cell has more than one solid neighbor its cell-centered velocity has to be parallel to all the non-parallel solid faces.

Furthermore, if the changes in density are to be taken into account, the condition on the cellcentered velocity normal to the solid faces has to be modified.

Due to these difficulties in the adoption of the internal solid boundary condition, another simpler approach is proposed in this work, which automatically adapts to the formulation with variable density. Here, the internal solid boundary condition for the pressure is "alleviated". Instead of directly imposing the solid boundary condition to the internal solid faces, the neighbor solid cell is used as a ghost cell for the pressure and introduced into the pressure system of equations.

Therefore, two categories of solid cells are defined: solid-solid and solid-liquid (see fig. 3). The former accounts for the solid cells whose neighbors are all also solid, while the latter contains the solid cells which have at least one liquid-containing neighbor cell. The solid-solid cells are the ones decoupled from the rest, enforcing zero filtration through their faces, while the solid-liquid cells are maintained in the system of pressure equations.

Therefore, the pressure equations for the solid-solid cells remain as those presented in [1]:

$$
a_{\mathrm{P}} \tilde{p}_{\mathrm{P}}^{n+1}=b_{\mathrm{P}}
$$

Since solid-liquid cells are maintained in the system of pressure equations, pressure is calculated in such cells as in the liquid-containing sub-system, enforcing mass conservation. The resulting pressure equations for these cells depend on the amount of liquid-containing neighbors. For example, for the 


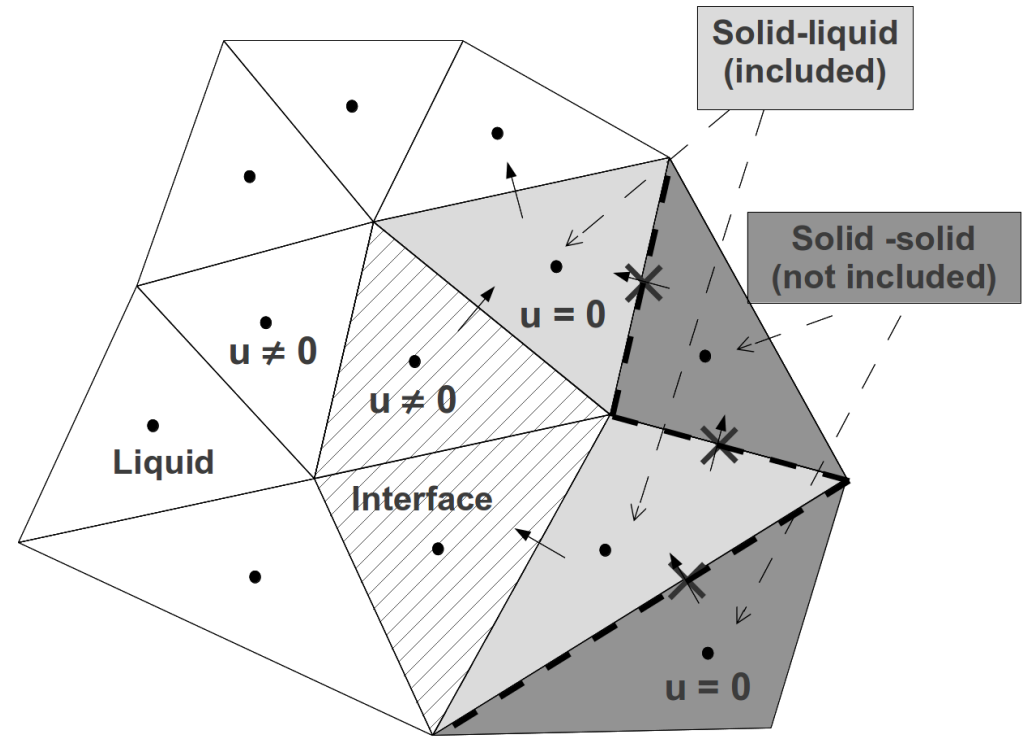

Figure 3: Sketch of unstructured mesh. White: liquid. Striped: interface. Light grey: solid-liquid cells (included in the non-trivial sub-system of pressure equations). Dark grey: solid-solid cells (trivial system of pressure equations).

solid-liquid cells with one and two neighbors, the equations remain:

One liquid neighbor:

$$
\begin{gathered}
\dot{m}_{f 1}^{n+1}=\frac{\dot{m}_{f 1}^{p}-\Delta t \nabla p^{n+1} \cdot \hat{n}_{f 1} A_{f 1}}{\left(1+\Delta t S / \rho_{l}\right)_{f 1}}=V_{\mathrm{P}} \frac{\rho_{m, \mathrm{P}}^{n+1}-\rho_{m, \mathrm{P}}^{n}}{\Delta t} \\
\Rightarrow a_{\mathrm{P}} \tilde{p}_{\mathrm{P}}^{n+1}+a_{\mathrm{F}_{f 1}} \tilde{p}_{\mathrm{F}_{f 1}}^{n+1}=b_{\mathrm{P}}
\end{gathered}
$$

Two liquid neighbors:

$$
\begin{gathered}
\dot{m}_{f 1}^{n+1}+\dot{m}_{f 2}^{n+1}=\sum_{f=f 1, f 2} \frac{\dot{m}_{f}^{p}}{\left(1+\Delta t S / \rho_{l}\right)_{f}}-\sum_{f=f 1, f 2} \frac{\Delta t \nabla p^{n+1} \cdot \hat{n}_{f} A_{f}}{\left(1+\Delta t S / \rho_{l}\right)_{f}}=V_{\mathrm{P}} \frac{\rho_{m, \mathrm{P}}^{n+1}-\rho_{m, \mathrm{P}}^{n}}{\Delta t} \\
\Rightarrow a_{\mathrm{P}} \tilde{p}_{\mathrm{P}}^{n+1}+a_{\mathrm{F}_{f 1}} \tilde{p}_{\mathrm{F}_{f 1}}^{n+1}+a_{\mathrm{F}_{f 2}} \tilde{p}_{\mathrm{F}_{f 2}}^{n+1}=b_{\mathrm{P}}
\end{gathered}
$$

It can be observed that, if there is no density change between consecutive time steps (i.e. the solid-liquid cell is solid in both), the mass flow through the cell face $f 1$ - connecting the solid and liquid-containing cells - resulting from Eq. (26) is zero. This means that the resulting pressure in the solid-liquid cell must be such that the mass flow through $f 1$ obtained by applying Eq. (20) is zero. On the other hand, if there is a change of density between successive time steps (e.g. a completely solid control volume starts to melt), the mass flow through the solid-liquid boundary is not zero anymore. Therefore, this internal boundary condition adapts itself automatically to the density variations.

In the cases of solid-liquid cells with two liquid-containing neighbors, Eq. (27) indicates that the mass fluxes through the solid-liquid faces $f 1$ and $f 2$ are not constrained to be strictly zero even if 


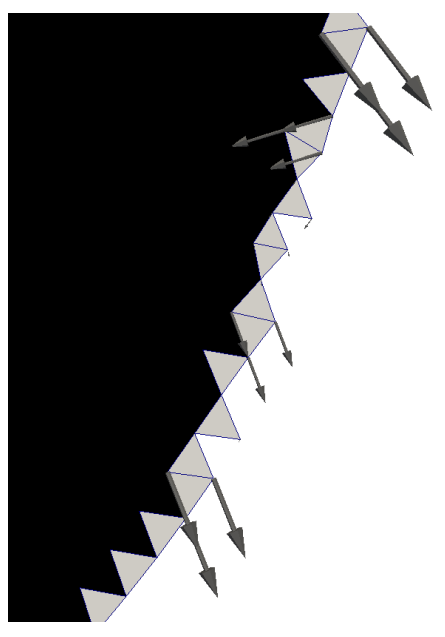

(a) Internal solid boundary approach

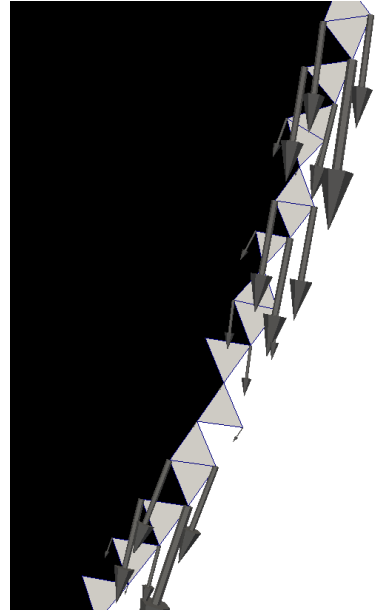

(b) Proposed approach

Figure 4: Velocity vectors of liquid-containing cells close to solid cells. Dark zone corresponds to the solid phase.

there is no variation of the density. Only their summation is required to be zero in such case, and therefore, it is possible for the liquid to "trespass" these solid-liquid cells. On the other hand, the cell-centered velocities in these special solid cells are explicitly set to zero, since no liquid is contained in them.

The proposed approach results in a "smoother" velocity field in the proximity of the solid cells, specially where completely liquid and solid cells are in direct contact (without an intermediate interface cell) in zones where the mesh resolution is not high. This can be seen in figure 4, showing the velocity vectors of the liquid-containing cells close to solid cells using both approaches, the one proposed in this work and the one imposing an internal solid boundary condition in the same way as in the mesh solid boundaries. It can be observed that in the latter case (fig. 4a) the velocity vectors are parallel to the faces of the solid cells, while in the former (fig. 4b) the fluid seems to go through them and the velocity field is less intrincate.

\subsubsection{Resolution of the linear system of pressure equations}

The resulting system of pressure equations has variable coefficients, where two sub-systems can be identified: one comprising all the liquid-containing and solid-liquid cells; and another, trivial one, with all the solid-solid cells where the value of pressure is not used.

Since the system of equations is symmetric, a preconditioned Conjugate Gradient (PCG) solver can be used. In order to lower the computations involved in the resolution of this system of equations, the PCG has been modified so as to perform matrix-vector multiplications only in the non-trivial sub-system, excluding the non-resolved solid-solid cells. 


\subsection{Energy}

The conservative form ( $\rho h$ as the calculated variable) of the energy equation is Eq. (3), which can be discretized in time as follows:

$$
(\rho h)_{m}^{n+1}=(\rho h)_{m}^{n}+\Delta t\left[-\nabla \cdot\left(\rho_{l} h_{l} \vec{u}\right)+\nabla \cdot\left(\lambda_{m} \nabla T\right)\right]^{n+\alpha}
$$

were the right hand side would be evaluated somehow depending on the time integration scheme.

It would be preferable to use this approach, since both density and enthalpy could be calculated at the same time. The key here is to be able to define the thermodynamic state from the value of $(\rho h)_{m}$, i.e. to calculate the rest of the properties $(T, \lambda$, etc. $)$ using $(\rho h)_{m}$ as the independent variable.

However, the usual situation is to have correlations for the properties expressed in terms of $T$, and furthermore, to have separate correlations for $\rho$ and $h$ (see section 2.1). Thus, in order to be able to use $\rho h$ to find the rest of the properties, one would have to multiply both correlations and afterwards invert the resulting formula to obtain $T$ from $\rho h$. This is usually a hard task, since could result in having to invert complicated functions.

Therefore, it is useful to be able to calculate only $h$ from the energy equation, while assuming $\rho$ as known. This can be done using the non-conservative form of the energy equation.

Taking into account the conversion:

$$
\frac{\partial \rho h_{m}}{\partial t}=h_{m} \frac{\partial \rho_{m}}{\partial t}+\rho_{m} \frac{\partial h_{m}}{\partial t}
$$

the following non-conservative form is obtained (where the calculated variable is $h$ ) from Eq. (3):

$$
\rho_{m} \frac{\partial h_{m}}{\partial t}+\nabla \cdot\left(\rho_{l} h_{l} \vec{u}\right)=\nabla \cdot\left(\lambda_{m} \nabla T\right)-h_{m} \frac{\partial \rho_{m}}{\partial t}
$$

and using mass conservation equation (1) it results in:

$$
\rho_{m} \frac{\partial h_{m}}{\partial t}+\nabla \cdot\left(\rho_{l} h_{l} \vec{u}\right)=\nabla \cdot\left(\lambda_{m} \nabla T\right)+h_{m} \nabla \cdot\left(\rho_{l} \vec{u}\right)
$$

Discretizing Eq. (29) in time, the following expression is obtained:

$$
h_{m}^{n+1}=h_{m}^{n}+\Delta t\left[\frac{-\nabla \cdot\left(\rho_{l} h_{l} \vec{u}\right)+\nabla \cdot\left(\lambda_{m} \nabla T\right)+h_{m} \nabla \cdot\left(\rho_{l} \vec{u}\right)}{\rho_{m}}\right]^{n+\alpha}
$$

Using this equation and those from section 2.1 , the thermodynamic state of the material can be found.

If there is an interest in using $T$ in the convective term instead of $h_{l}$, the conversion $d h=C_{p} d T$ can be used, which applied into the following non-conservative form of the energy equation: 


$$
\rho_{m} \frac{\partial h_{m}}{\partial t}+\nabla h_{l} \cdot \rho_{l} \vec{u}=\nabla \cdot\left(\lambda_{m} \nabla T\right)+\left(h_{m}-h_{l}\right) \nabla \cdot\left(\rho_{l} \vec{u}\right)
$$

results in:

$$
\rho_{m} \frac{\partial h_{m}}{\partial t}+C_{p_{l}} \nabla T \cdot \rho_{l} \vec{u}=\nabla \cdot\left(\lambda_{m} \nabla T\right)+\left(h_{m}-h_{l}\right) \nabla \cdot\left(\rho_{l} \vec{u}\right)
$$

In order to express the convective term as a divergence, the following identity can be used:

$$
C_{p_{l}} \nabla T \cdot\left(\rho_{l} \vec{u}\right)=C_{p_{l}} \nabla \cdot\left(\rho_{l} \vec{u} T\right)-C_{p_{l}} T \nabla \cdot\left(\rho_{l} \vec{u}\right)
$$

to obtain:

$$
\rho_{m} \frac{\partial h_{m}}{\partial t}+C_{p_{l}} \nabla \cdot\left(\rho_{l} \vec{u} T\right)=\nabla \cdot\left(\lambda_{m} \nabla T\right)+\left(h_{m}-h_{l}+C_{p_{l}} T\right) \nabla \cdot\left(\rho_{l} \vec{u}\right)
$$

where there is an extra term in the right hand side of the equation depending on the mass balance in the control volume (which, in general, will be different from zero due to the changes in the density), the total enthalpy and the difference between the liquid enthalpy and the liquid specific heat multiplied by the temperature.

Since $h_{l}$ appears in the source term, it has to be calculated even if it is not appearing in the advective term. Therefore, not using $h_{l}$ in the advective term would only be interesting if there is some advantage in terms of computational cost. It is possible that some advantage could be obtained if, for example, the calculation of convective and diffusive terms is performed at the same time, and using a unique array/vector of values $(T)$ could turn out faster - due to more efficient memory management - than needing two different arrays/vectors $\left(T\right.$ and $\left.h_{l}\right)$.

Discretizing Eq. (31) in time, the following expression is obtained:

$$
h_{m}^{n+1}=h_{m}^{n}+\Delta t\left[\frac{-C_{p, l} \nabla \cdot\left(\rho_{l} \vec{u} T\right)+\nabla \cdot\left(\lambda_{m} \nabla T\right)+\left(h_{m}-h_{l}+C_{p_{l}} T\right) \nabla \cdot\left(\rho_{l} \vec{u}\right)}{\rho_{m}}\right]^{n+\alpha}
$$

\section{Definition of the case study}

The case study is based on the experimental and numerical results obtained by $[16,27]$, where the melting of a PCM inside a spherical capsule is studied. Figure 5 depicts a sketch of the configuration of the case. A spherical glass capsule filled with n-Octadecane, initially in solid state, is immersed into a water bath at $40^{\circ} \mathrm{C}$. Since the temperature of the water is higher than the melting point of the PCM, the material close to the capsule shell starts to melt forming a concentric solid-liquid interface. As the melting process evolves, the liquid layer grows and the temperature gradients in the fluid combined with buoyancy forces cause the liquid to move, producing the natural convection phenomenon. This form of heat transfer intensifies the heat transfer rate and modifies the concentrical heat transfer that 


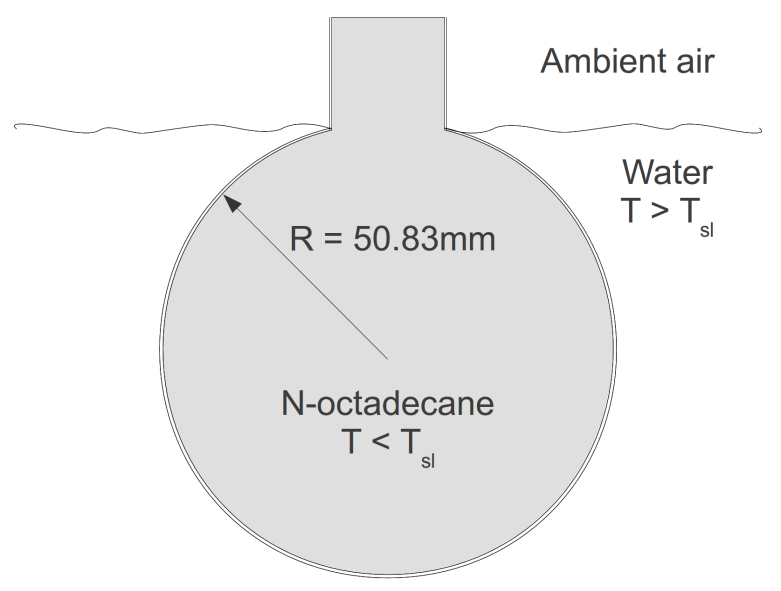

Figure 5: Sketch of the case under study

was enforced by the thermal conduction. Hence, a deformation of the interface is produced, since its evolution is determined by the heat transfer rate.

In [27], experiments of constrained and unconconstrained melting are presented. In unconstrained melting, the solid phase, being denser than the liquid phase, sinks to the bottom of the sphere; while in constrained melting, the solid phase is restrained from sinking. Here, numerical simulations of constrained melting are presented, since the solid phase is assumed to have zero velocity.

Both two and three-dimensional meshes have been used, which were generated with ANSYS ICEM CFD package [28]. Table 1 shows the list of meshes used, where a code for each one has been assigned indicating if the mesh is two (2D) or three-dimensional (3D), if it includes or not the upper opening (NO: No Opening; wo: With Opening) and the approximate amount of cells. The upper opening was included in the meshes intended to be used for simulating cases with variable density, where the less dense fluid needs to be allowed to escape from the capsule.

In the case of $2 \mathrm{D}$ meshes, since a cartesian formulation is used, the geometry used is a slice of sphere with a very small azimuthal angle (see left half of figs. 6a-6d ). It should be noted that the 2D meshes 2D-NO-7.7E3 and 2D-WO-9.3E3 (without counting the cells of the upper opening) have a similar amount of cells as that claimed to be used by Tan et al. [16] in their simulations $(\sim 7000-8000)$, while meshes 2D-NO-31E3 and 2D-WO-31E3 have a density of around 4 times higher. 3D meshes 3DNO-1.4E6 and 3D-WO-1.6E6 present a density of cells for a slice of sphere (similar to the geometry of the $2 \mathrm{D}$ meshes used) of around 5000 cells — somewhat lower than 2D-NO-7.7E3 and 2D-WO-9.3E3while for mesh 3D-WO-5E6 (only used in case C) this value is around 12000.

\subsection{Spatial discretization schemes}

A central difference scheme has been used for the diffusive terms of both momentum and energy equations. The convective term of momentum equation has been discretized with a symmetry- 
Table 1: Meshes used

\begin{tabular}{cccccc}
\hline \multirow{2}{*}{ Code } & \multirow{2}{*}{ Cells } & Cells for opening & Prismatic layers & \multicolumn{2}{c}{$r_{e q} / R$} \\
\cline { 5 - 6 } & & & & Smallest $^{1}$ & Biggest $^{1}$ \\
\hline 2D-NO-7.7E3 & 7669 & - & - & $4.28 \mathrm{E}-3$ & $3.91 \mathrm{E}-2$ \\
2D-NO-31E3 & 30670 & - & - & $2.03 \mathrm{E}-3$ & $2.03 \mathrm{E}-2$ \\
2D-WO-9.3E3 & 9257 & 400 & - & $2.89 \mathrm{E}-3$ & $2.03 \mathrm{E}-2$ \\
2D-WO-31E3 & 31445 & 400 & 10 & $8.56 \mathrm{E}-4$ & $1.64 \mathrm{E}-2$ \\
3D-NO-1.4E6 & 1436919 & - & - & $4.91 \mathrm{E}-3$ & $1.99 \mathrm{E}-2$ \\
3D-WO-1.6E6 & 1637607 & 12700 & - & $3.44 \mathrm{E}-3$ & $9.42 \mathrm{E}-3$ \\
3D-WO-5E6 & 5272390 & $\sim 40000$ & &
\end{tabular}

${ }^{a}$ Equivalent radius of the biggest and smallest cells. In $2 \mathrm{D}$ meshes, it is equal to the radius of the circle with the same area as the biggest/smallest cell; while in $3 \mathrm{D}$ meshes it is equal to the radius of the sphere with the same volume. All values are relative to the radius of the spherical capsule.

preserving scheme (SYMMPRES) [29]. This scheme considers the scalars in the cell faces as the mean value between the scalars in the cell centers. In uniform meshes its accuracy is of second order, while in unstructured meshes, although locally first order, it has been observed to be of a higher order (between 1 and 2) globally [30].

For the convective term of the energy equation two schemes were tested, a first-order UPWIND and the symmetry preserving scheme. The former has the advantage of avoiding unbounded values of the temperature, while the latter has the property of presenting a lower numerical diffusion. A comparison between the results obtained with both schemes is carried out in the results section.

\subsection{Physical properties}

Difficulties have been encountered in the search of the physical properties of n-Octadecane near the melting point. Specially for density of the solid phase and thermal conductivity of both phases, as well as for the fusion temperature. Little information, and sometimes, high disagreements between the different sources has been found.

\subsubsection{Constant properties}

As a first approximation, thermo-physical properties were taken from [16]. The significant quantitative difference between experimental and first numerical results led to the suspicion of being using wrong properties. Therefore, a thorough search for physical properties has been carried out. Very 


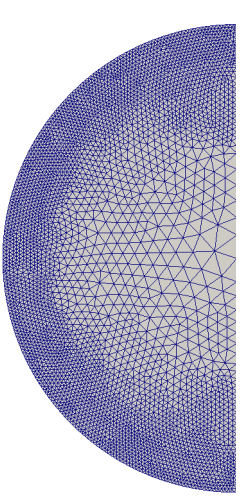

(a) 2D-NO-7.7E3

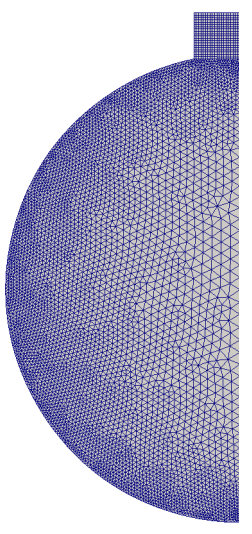

(c) 2D-WO-9.3E3

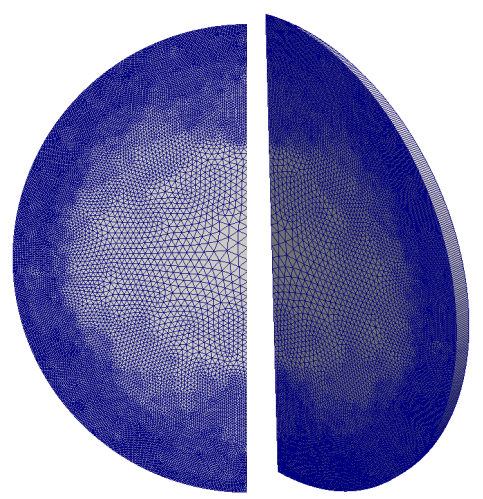

(b) 2D-NO-31E3

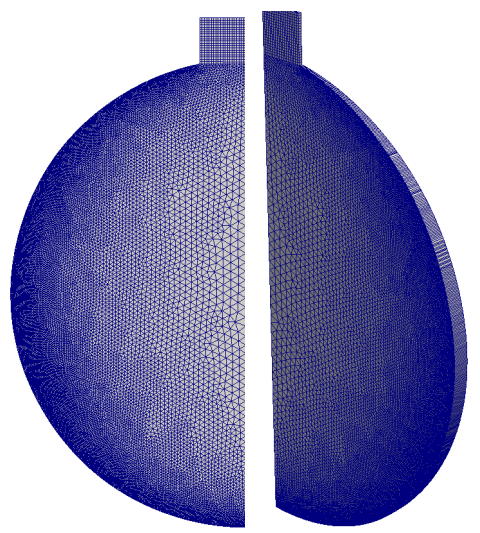

(d) 2D-Wo-31E3

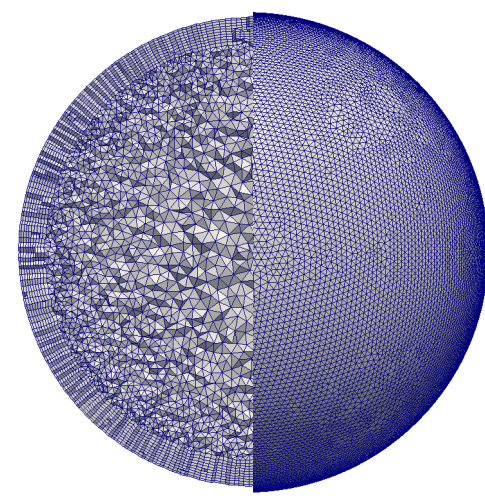

(e) 3D-NO-1.4E6

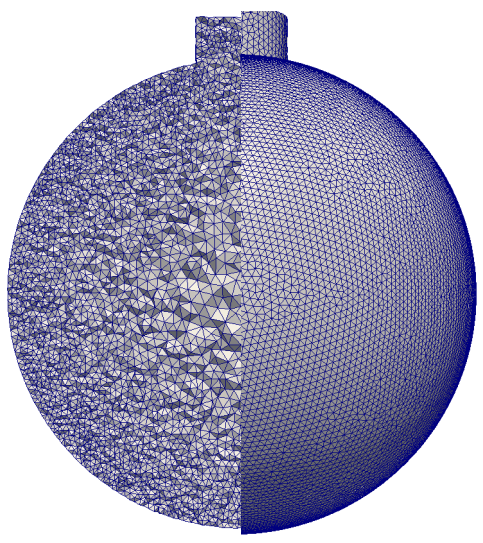

(f) $3 \mathrm{D}-\mathrm{WO}-1.6 \mathrm{E} 6$

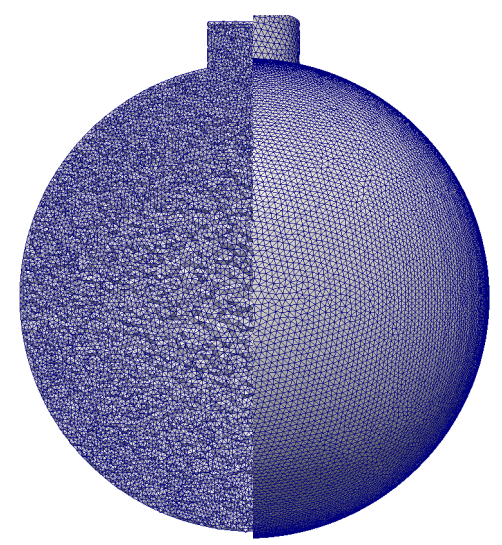

(g) 3D-WO-5E6

Figure 6: Meshes used. 2D: Left half of images show a front view, while right half show a tilted view. 3D: Left half of images show a front cut view, while right half show the external surface.

little information has been found for some of them, such as density and thermal conductivity of the solid phase.

Table 2 depicts the numerical properties obtained from [16] and those generated using the data found in the literature $[31,32,33,34,35,36,37,38]$. 
Table 2: Constant physical properties of n-Octadecane

\begin{tabular}{|c|c|c|c|c|c|c|}
\hline & $\begin{array}{c}L \\
{\left[J k g^{-1}\right]}\end{array}$ & $\begin{array}{c}\rho \\
{\left[k g ~ m^{-3}\right]}\end{array}$ & $\begin{array}{c}C_{p} \\
{\left[J k g^{-1} K^{-1}\right]}\end{array}$ & $\begin{array}{c}\lambda \\
{\left[W m^{-1} K^{-1}\right]}\end{array}$ & $\begin{array}{c}\mu \\
{[P a s]}\end{array}$ & $\begin{array}{c}\beta \\
{\left[K^{-1}\right]}\end{array}$ \\
\hline From Tan et al. [16] & 243500 & 772.0 & 2300 & 0.1505 & $3.86 \mathrm{E}-3$ & $9.10 \mathrm{E}-4$ \\
\hline $\begin{array}{l}\text { From }[31,32,33,34, \\
35,36,37,38]\end{array}$ & 242454 & 774.5 & 2225 & 0.1505 & $3.57 \mathrm{E}-3$ & $9.42 \mathrm{E}-4^{1}$ \\
\hline $\begin{array}{l}\text { Proposed in this work } \\
(L \text { modified })\end{array}$ & 270159 & 774.5 & 2225 & 0.1505 & $3.57 \mathrm{E}-3$ & $9.42 \mathrm{E}-4^{1}$ \\
\hline
\end{tabular}

${ }^{a}$ Calculated as $\frac{\rho_{l}(313.15 K)-\rho_{l}(301.33 K)}{774.5 \frac{\mathrm{kg}}{\mathrm{m}^{3}} 11.82 K}$ using the correlation for liquid density from [34].

An important issue to point out is that the value of latent heat adopted in our simulations is not exactly the one found in [31], but a correction of that value considering the fact that the density of the liquid phase is being adopted for both phases. Thus, the value of $L$ is augmented by the ratio between solid and liquid densities $\left(\rho_{s} / \rho_{l}\right)$ in order to obtain the real latent heat inside each control volume. Therefore,

$$
L_{\text {mod }}=242454 \frac{\rho_{s}}{\rho_{l}}=270159 \mathrm{~J} / \mathrm{kg} ; \quad \text { with: } \rho_{s}=863 \mathrm{~kg} / \mathrm{m}^{3}
$$

If the original value of $L$ were used together with a density equal to that of the liquid phase, less energy than that actually needed would be required to melt the solid contained in the same control volume, and therefore, a faster melting would be obtained. This issue has not been addressed by other authors (e.g. $[16,17])$, and may have been a source of error in their numerical simulations.

In this case, adopting the real latent heat or the modified one results in that around $11 \%$ more energy is needed to melt the n-Octadecane contained in the same control volume for the latter case. This is one possible cause for having melting rates higher than those registered in the experiment, by the numerical simulations of Tan et al. [16] and Zhao et al. [17].

\subsubsection{Variable properties}

In the pursue of accurate thermo-physical properties of n-Octadecane, several works have been consulted (several more than those referred in this work). Discrepancies have been found for some properties between different works. The correlations finally adopted are presented below, along with comments about their accuracy and some other related observations.

- Melting temperature $\left(T_{s l}\right)$ : taken from the experimental work of Messerly et al. [31]. A fixed value 
of $301.33 \mathrm{~K}\left(28.18^{\circ} \mathrm{C}\right)$ has been adopted. Several references $[32,39]$ indicate that n-Octadecane does not present disordered phases between the low temperature ordered solid phase and the liquid phase, resulting in a change of phase at a single temperature. There is some disagreement about this value, and melting points as low as $300.2 \mathrm{~K}$ have been reported [33]. Furthermore, it has been seen in the scientific literature (e.g. in [32]) that impurities could modifiy the behaviour of pure n-Octadecane resulting in a modification of the melting temperature and of the latent heat of fusion. It is believed, due to the experimental temperature curves presented in [16] that the material used in the experiments could present a melting temperature range within $27-29^{\circ} \mathrm{C}$. This could definitely be a cause of differences between experimental and numerical results. With this in mind, numerical simulations using a melting point of $300.35 \mathrm{~K}\left(27.2^{\circ} \mathrm{C}\right)$ are also carried out in this work.

- Latent heat of fusion $(\mathrm{L}): 242454 \mathrm{~J} / \mathrm{kg}$, calculated from the liquid and solid enthalpies at $301.33 \mathrm{~K}$ indicated in [31]. As mentioned above, this value could be modified by the presence of impurities.

- Liquid density $\left(\rho_{l}\right)$ : correlation adopted from [34],

$$
\rho_{l}=1010.07-0.80587 T+0.00012463 T^{2}
$$

where $\rho_{l}$ is expressed in $\mathrm{kg} / \mathrm{m}^{3}$ and the temperature is in $K$. The variation of this value in the working range of temperatures is around $1 \%$ using the above correlation.

Although the authors of the referred work define a range of validity of their correlation which do not include the temperature range between the melting point and the maximum temperature present in the experiment of Tan et al. [16], comparison with the experimental curve presented in [35] shows a fairly good agreement for this range of temperatures.

- Liquid viscosity $\left(\mu_{l}\right)$ : correlation adopted from [36],

$$
\log _{10} \mu_{l}=-11.5505+\frac{1670.8}{T}+0.015675 T-1.2341 \times 10^{-5} T^{2}
$$

where $\mu_{l}$ is expressed in Pa.s and the temperature is in $K$.

This correlation reproduce almost exactly the experimental values from [40] in the range of temperatures of interest. The variation of this property is not negligible in the working range of temperatures of the liquid phase $(\sim 30 \%)$. 


$$
\lambda_{l}=0.156427-0.0001841779(T-273.15)
$$

where $\lambda_{l}$ is expressed in $W / m . K$ and the temperature is in $K$. The variation of this property in the working range of temperatures, using the above expression, is less than $2 \%$.

Differences of around $5 \%$ have been found with those values calculated using the correlation from [36], and higher with respect to [38]. In the latter work, a value of liquid thermal conductivity of $0.18 \mathrm{~W} / \mathrm{mK}$ ( $20 \%$ higher than that obtained with the present correlation) has been reported at the melting point.

- Liquid specific heat $\left(C_{p, l}\right)$ : linearized correlation from [37],

$$
C_{p, l}=2137.1456+2.7186(T-273.15)
$$

where $C_{p, l}$ is expressed in $J / k g . K$ and the temperature is in $K$. The variation of this property in the working range of temperatures, using the above expression, is less than $2 \%$.

The present correlation agrees very well with the experimental data from [31]. Differences of up to $10 \%$ can be found with the values calculated with the correlation from [39], which is seen to underpredict the values in the temperature range between $300-320 \mathrm{~K}$.

- Solid density $\left(\rho_{s}\right)$ : a value of $863 \mathrm{~kg} / \mathrm{m}^{3}$ has been adopted. There is a high uncertainty in this value, as no exact information has been found. It has been very roughly approximated from the density curve presented in [35].

There is a significant difference between the densities of the liquid and solid phases. With the values adopted in this work, at the melting point, this difference is of $\sim 11 \%$ (with respect to the liquid density).

- Solid thermal conductivity $\left(\lambda_{s}\right)$ : a fixed value of $0.3362 \mathrm{~W} / \mathrm{m} . \mathrm{K}$ has been adopted, calculated from the ratio $k_{s} / k_{l}$ presented in [38] and from $k_{l}$ (at the melting point) determined using the previously indicated correlation. There is high uncertainty in this value as high dispersion has been found between different sources consulted. The authors believe that the ratio $k_{s} / k_{l}=0.45$ is the most accurate value of those found in [38] due to the procedure used for its determination, which do not depend on the density of any of the phases. However, it is believed that there is a somewhat linear variation of this property with the temperature, as seen for other n-alkanes [41]. Unfortunately, no correlation has been found for the n-Octadecane. 
In any case, there is a significant difference between the thermal conductivities of the liquid and solid phases, which could be a source of error in the numerical simulations if not properly taken into account.

- Solid specific heat $\left(C_{p, s}\right)$ : linearized experimental data from [31],

$$
C_{p, s}=1652.9056+10.2673(T-273.15)
$$

The above expression results in a variation of less than $1 \%$ of this property in the working range of temperatures for the solid phase. However, there is a significant difference between the specific heats of the solid and liquid phases, which is around $13 \%$.

The correlation presented in [39] has not been adopted here due to not being very accurate in the temperature range near the melting point, as indicated by the authors.

\subsection{Initial and boundary conditions}

\subsubsection{Initial conditions}

Initially, all the PCM is in its solid state. However, in order to avoid the formation of liquid cells completely surrounded by solid cells — which would cause divergence, due to the impossibility of the less dense fluid to leave these cells - a liquid film close to the shell has been included in the initial conditions. The width of this layer is around $3 \%$ of the radius of the capsule $(\sim 1.5 \mathrm{~mm})$, resulting in an initial total liquid fraction of $\sim 8.5 \%$, depending on the mesh. Therefore, all numerical simulations in this work are assumed to start in a state corresponding to that of the experiment at $\mathrm{t}=12$ minutes, which seems to be the time at which liquid fraction is around $\sim 8.5 \%$, according to the figures presented by $[16]$.

For the liquid film, a temperature equal to the melting point has been adopted. For the solid phase, a uniform temperature of $26.5^{\circ} \mathrm{C}$ has been assumed, estimated from the experimental temperature curves presented in [16].

\subsubsection{Boundary conditions}

- Capsule shell In the numerical results reported by Tan et al. [16], the melting rate was overpredicted when compared to the experimental data. They justified the sources for such discrepancy in the consideration of a constant temperature for the external surface of the capsule shell, when the presence of the base supporting the capsule could be avoiding a good heat transfer in this zone, and furthermore, a thermal stratification could have developed in the water bath. With this in mind, instead of adopting a constant temperature for the outer surface of the capsule, 
a convection coefficient acting between the water bath and the shell is assumed. In fact, two values of this coefficient are tested, one considering a high heat transfer rate between the water bath and the capsule and another one with a low heat transfer rate.

Therefore, the capsule shell is represented as a solid, no-slip boundary (see section 3.2.2), with a fixed external temperature $\left(40^{\circ} \mathrm{C}\right)$ and an equivalent heat transfer coefficient $\left(\alpha_{e q}\right)$ encompassing thermal convection between the bath and the outer surface and conduction through the glass, respectively. A heat flux into the cell adjacent to the capsule is enforced as:

$$
\dot{q}_{s b}=A_{s b} \frac{\left(T_{w b}-T_{P}\right)}{\frac{A_{s b}}{A_{e x t} \alpha_{e q}}+\frac{d_{P, s b} \cdot \hat{n}_{s b}}{\lambda_{f}}}
$$

where the thermal resistance has been indicated as a sum of external (bath + shell) plus internal (portion of fluid between cell center and solid face) heat transfer resistance. The two values of $\alpha_{e q}$ here adopted are $796 \mathrm{~W} /\left(\mathrm{m}^{2} \mathrm{~K}\right)$ and $237 \mathrm{~W} / \mathrm{m}^{2} \mathrm{~K}$. The former corresponds to a convective heat transfer between bath and shell of around $3000 \mathrm{~W} / \mathrm{m}^{2} \mathrm{~K}$, while the latter corresponds to a convective coefficient of around $300 \mathrm{~W} / \mathrm{m}^{2} \mathrm{~K}$. The thickness of the glass capsule shell is $1.5 \mathrm{~mm}$. Actually, what is really imposed as a thermal boundary condition is the temperature of the boundary face, which is determined in order to give the previous heat flux into the cell, as follows:

$$
T_{s b}=\frac{\frac{A_{e x t} \alpha_{e q}}{A_{s b}} T_{w b}+\frac{\lambda_{f}}{d_{P, s b} \cdot \hat{n}_{s b}} T_{P}}{\frac{A_{e x t} \alpha_{e q}}{A_{s b}}+\frac{\lambda_{f}}{\overrightarrow{d_{P, s b} \cdot \hat{n}_{s b}}}}
$$

- Upper opening (variable density cases)

Fluid flow is allowed to go out from (or into) the capsule through this upper opening. For this, a condition on pressure is applied, resembling a Bernoulli equation, in the form:

$$
p_{f b}=p_{\infty}-\gamma \frac{\rho u^{2}}{2}
$$

where $\gamma$ is a coefficient controlling the loss of kinetic energy, which can take values from 0 to 1. In the cases presented, values of $p_{\infty}=0$ and $\gamma=0.5$ have been used. The velocities of the boundary faces are assumed to be equal to those of the corresponding cell nodes.

For the temperature, in the boundary faces through which the flow goes out from the capsule, a zero derivative has been assumed, taking the value of the cell. For the boundary faces through which the flow comes into the capsule, the temperature of the incoming flow has been assumed 
to be the mean value (weighted by the mass flow) of the temperature corresponding to the flow going out from the capsule. This results in a zero overall transport of thermal energy through the opening.

For the lateral surface of the upper opening — which is part of the capsule shell- the same heat flux condition is used as for the rest of the shell, in $2 \mathrm{D}$ cases. However, in $3 \mathrm{D}$ cases, a further improvement has been introduced by modeling the fact that this part of the shell is in contact with the outer air instead of with the water bath, as it is apparent in the pictures of the experiments presented in [27]. For this zone, in 3D cases, a value of $5.1 \mathrm{~W} / \mathrm{m}^{2}$ has been adopted for $\alpha_{e q}$ and $25^{\circ} \mathrm{C}$ for the external (air) temperature.

- Periodic faces (2D)

For carrying out 2D simulations, where axi-symmetry is assumed, the faces of the sphere slices (see 2D meshes from fig. 6) should not affect the solution in any way. Therefore, zero derivative has been adopted for all the variables, as well as zero mass flux through the faces. No contribution of these boundary faces is produced over the diffusive, convective or source terms of all the equations.

\section{Results}

Several simulations have been run using the different meshes designed, for two and three-dimensional analyses, as well as using constant and variable thermo-physical set of properties. Since the thermal boundary conditions are not exactly known, two different values have been tested for the equivalent heat transfer coefficient between the water thermal bath and the internal surface of the capsule shell $\left(\alpha_{e q}=796 \mathrm{~W} / \mathrm{m}^{2} \mathrm{~K}\right.$ and $\left.\alpha_{e q}=237 \mathrm{~W} / \mathrm{m}^{2} \mathrm{~K}\right)$. Furthermore, since the experimental temperature curves observed in [16] indicate that the onset of the phase change may be occurring at a temperature of around $27^{\circ} \mathrm{C}$, simulations assuming a melting point of $27.2^{\circ} \mathrm{C}$ have also been run. Three combinations of thermal boundary condition and melting point are codified as cases A, B and C, as depicted in Table 3.

Comparison is performed between simulations using UPWIND and SYMMPRES convective schemes, two and three-dimensional meshes and constant and variable properties. Agreement with experimental results is also addressed.

\subsection{Convective scheme and grid convergence}

As indicated in section 4.1, two schemes have been adopted for the discretization of the convective term of the energy equation, first-order UPWIND and SYMMPRES. 
Table 3: Cases run using different combinations of boundary conditions and melting point

\begin{tabular}{ccc}
\hline Code & $\alpha_{e q}\left(W / m^{2} K\right)$ & $T_{s l}\left({ }^{\circ} \mathrm{C}\right)$ \\
\hline $\mathrm{A}$ & 796 & 28.18 \\
$\mathrm{~B}$ & 237 & 28.18 \\
$\mathrm{C}$ & 237 & 27.2 \\
\hline
\end{tabular}

Figure 7 show results of evolution of global liquid fraction for the different cases using each convective scheme. A better match with experimental results is observed in the results using the symmetry preserving scheme. Furthermore, the discrepancy between results with different meshes is higher in the cases using the UPWIND scheme, indicating a worse grid convergence. The differences between results using constant and variable properties are also higher in the cases where the UPWIND scheme was adopted.

All these observations indicate that the numerical diffusion is affecting the cases were the UPWIND scheme was used and that the symmetry preserving scheme seem to give more accurate results. Unbounded values of temperature have been observed at some specific locations in the SYMMPRES cases, but this problem does not seem to affect the results as much as the numerical diffusion produced by the UPWIND scheme. For this reason, the results presented in the following sections correspond to the simulations using the SYMMPRES scheme.

As the mesh density is increased, the melting rate tends to decrease. This behavior is observed for all the combinations of properties and boundary conditions tested. With the meshes used, it cannot be stated that grid convergence has been reached, although the results obtained with the SYMMPRES cases show a quite low disparity between them.

\subsection{Thermo-physical properties}

As indicated in section 4.2.1, when defining the constant thermo-physical properties used representative of the material in the working conditions, a modification to the latent heat was performed in order to correct the error in total energy; which comes from the fact of not considering the difference in density between liquid and solid phases.

Figure 8 shows the difference in liquid fraction evolution between three 2D simulations of case A, using different set of properties. For the first, constant properties shown in Table 2, without the modification of $L$, were adopted. In the second, constant properties with the modified $L$ were used. In the third, different densities and specific heats for liquid and solid phases are adopted (and therefore 


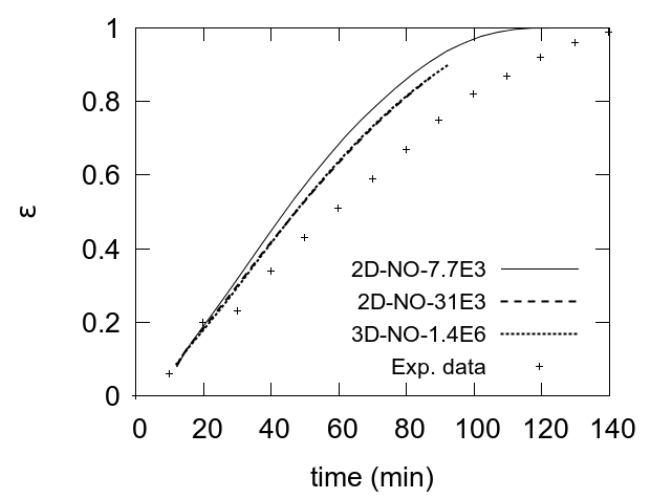

(a) Case A. Constant properties. UPWIND scheme.

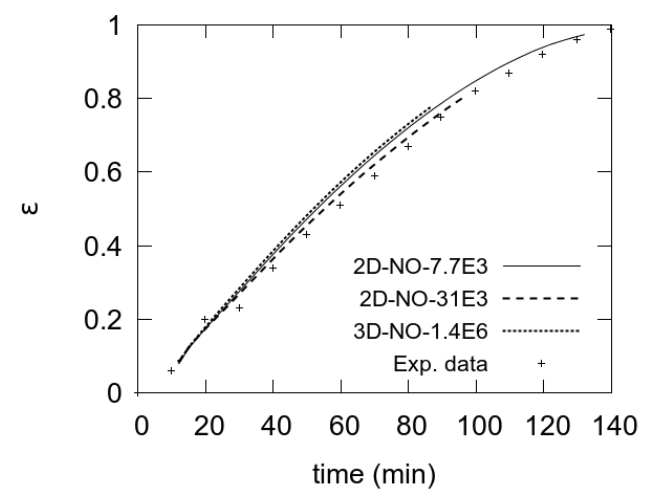

(c) Case A. Constant properties. SYMMPRES scheme.

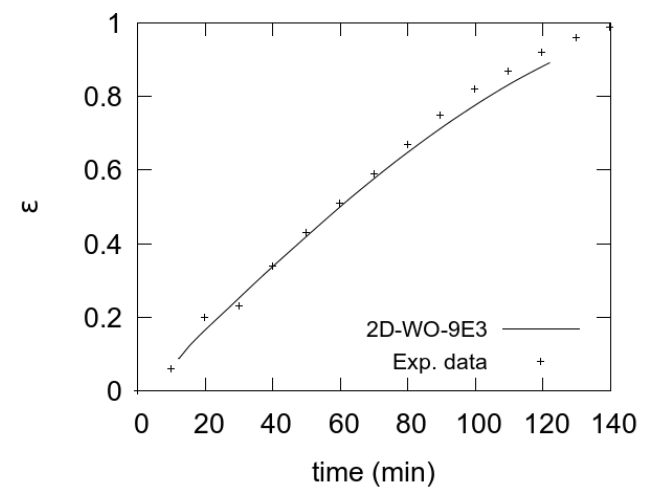

(e) Case B. Variable properties. SYMMPRES scheme.

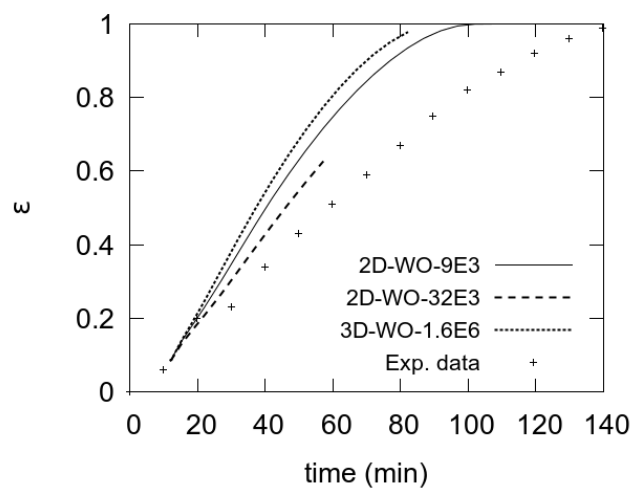

(b) Case A. Variable properties. UPWIND scheme.

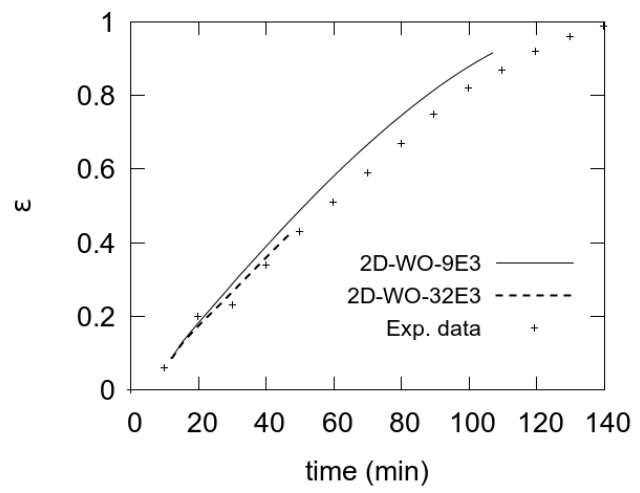

(d) Case A. Variable properties. SYMMPRES scheme.

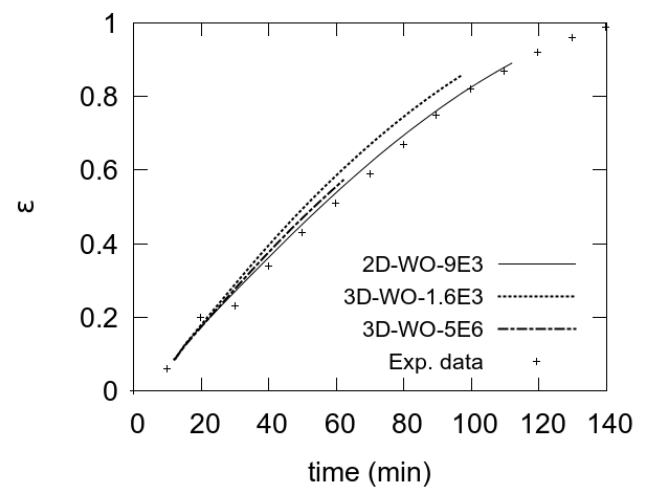

(f) Case C. Variable properties. SYMMPRES scheme.

Figure 7: Global volumetric liquid fraction vs. time for the different cases with 2D and 3D meshes, using upwind or symmetry preserving schemes.

a mesh with the upper opening is used for allowing fluid escaping from the shell) but with the rest of the properties constant. $\rho=863 \mathrm{~kg} / \mathrm{m}^{3}$ and $C_{p}=1940 \mathrm{~J} / \mathrm{kgK}^{-1}$ were used for the solid phase. Both simulations using constant properties were run with the same mesh, while for the other a mesh with 
a similar amount of cells was adopted.

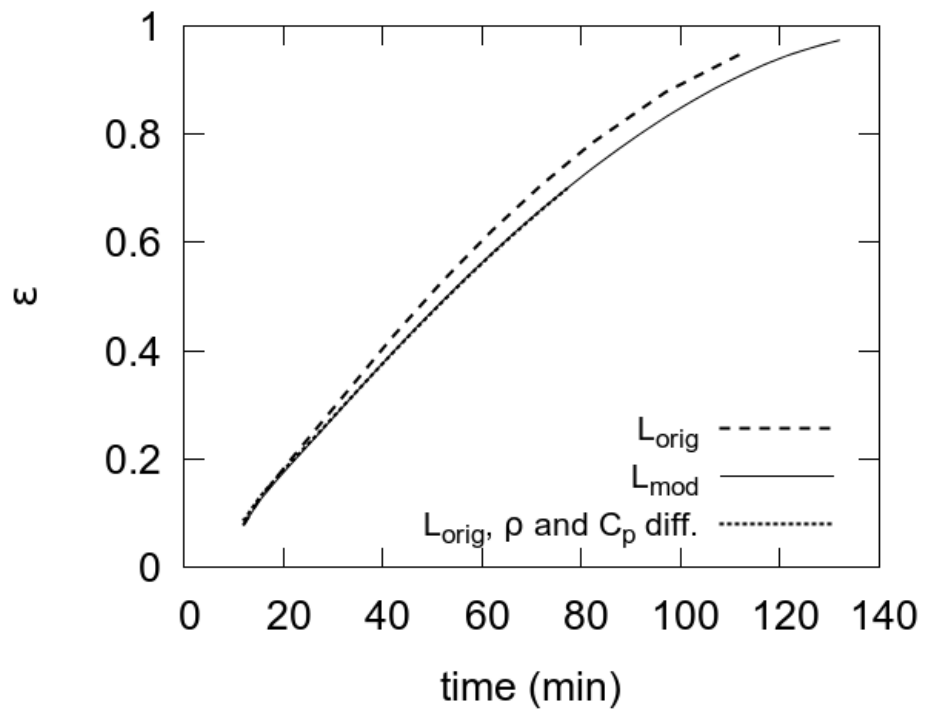

Figure 8: Numerical results of the evolution of global $\epsilon$ with time for case $\mathrm{A}$. $\mathrm{L}_{\text {orig }}$ : constant properties, unmodified value of $L$; mesh: 2D-NO-7.7E3. $\mathrm{L}_{\text {mod }}$ : constant properties, modified value of $L$; mesh: 2D-NO7.7E3. $\mathrm{L}_{\text {orig }}, \rho$ and $\mathrm{C}_{\mathrm{p}}$ diff.: different densities and specific heats for liquid and solid phases with the rest of the properties constant; mesh: 2D-WO-9.3E3.

It can be observed that the melting rate in the case with the unmodified $L$ is higher than for the other two cases, and that the case with constant properties and modified $L$ evolves almost exactly as the one where the differences in $\rho$ and $C_{p}$ are considered. The melting rates - between 30 and 80 minutes - for cases with constant properties and the original and modified $L$, result in values of $0.95 \% / \mathrm{min}$ and $0.88 \% / \mathrm{min}$, respectively; which is a decrease of around $8 \%$ of the melting rate, produced by the increase of around $11 \%$ in $L$. These observations indicate that the density scaling of $L$ compensates the fact of using the liquid density for both phases, and that the effect of the expansion in the melting is not very significant for this case.

Furthermore, plots of the evolution of the global liquid fraction of two 2D simulations, one using constant properties and another taking full account of the properties variations with temperature, are depicted in figure 9. It is observed that the consideration of the change of properties with the temperature result in a slight increase of the melting rate $(0.90 \% / \mathrm{min})$; being around $2-3 \%$ higher than in the case with constant properties and modified L. Further analysis, after running several simulations changing the property for which the variations with temperature are accounted for, seem to indicate that the property whose variation is affecting the melting rate the most is the thermal conductivity, and specially that of the liquid phase. 


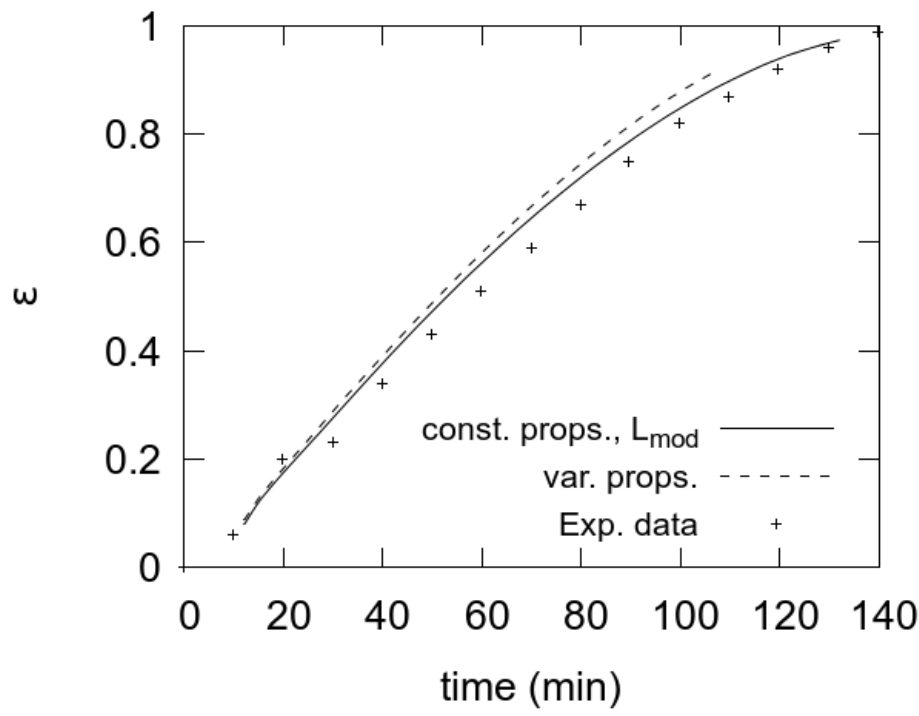

Figure 9: Numerical results of the evolution of global $\epsilon$ with time for case A using constant and variable properties. Simulation with constant, modified, properties (solid line) was run using mesh 2D-NO-7.7E3, while the one with variable properties (dashed line) was run with mesh 2D-WO-9.3E3.

\section{3. $2 D$ vs. $3 D$}

Figures 10 and 11 show the temperature and liquid fraction maps, as well as the streamlines of $2 \mathrm{D}$ and $3 \mathrm{D}$ simulations for two different moments corresponding to the experiment after $\mathrm{t} \sim 22$ and t $\sim 47$ minutes, respectively. Results shown correspond to meshes with a similar density (comparing the $2 \mathrm{D}$ mesh with a slice of the 3D mesh) in order to evaluate only the effect of assuming (or not) the hypothesis of axi-symmetric behaviour.

The common feature in two and three-dimensional results is the upgoing hot flow close to the shell and downgoing cold flow close to the solid, produced by the gravity force in combination with a decrease of density in the liquid as its temperature increases. However, there are differences between the flow patterns encountered in 2D and 3D cases.

In the bottom, due to the unstable thermal layer (hot shell below cold solid), the flow has a highly 3D behaviour. While in 2D cases the fluid eddies formed at the bottom are "trapped" by the fluid eddies located at the top/middle of the capsule (see 2D streamlines in figs. 10 and 11), in 3D cases the high velocity flows generated at the bottom "find their way" to the top flowing close to the shell (where the temperature is higher), through some periodically arranged azimuthal angles which alternate with those angles through which the cold fluid flows downwards (closer to the solid, cold, phase). Therefore, upward and downward flows show higher and lower velocities in alternate azimuthal angles, as seen in Fig. 12. Also in this figure it can be observed how the interface is modified due to this phenomenon. Figure 13 shows some temperature maps of the capsule shell, seen from below, where the alternating 


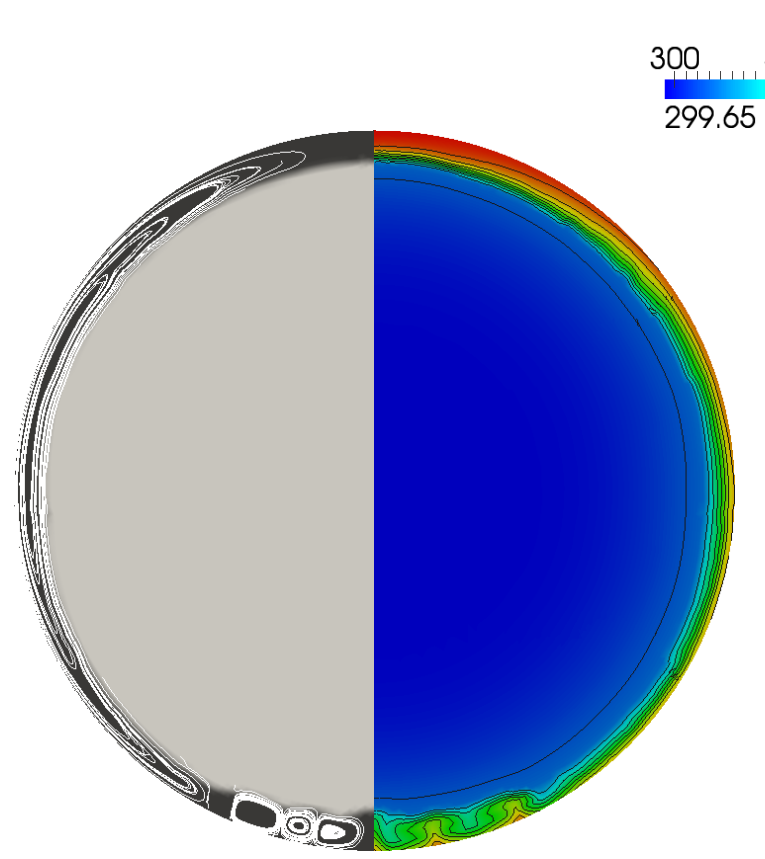

(a) Mesh: 2D-NO-7.7E3. Case A. Constant properties.

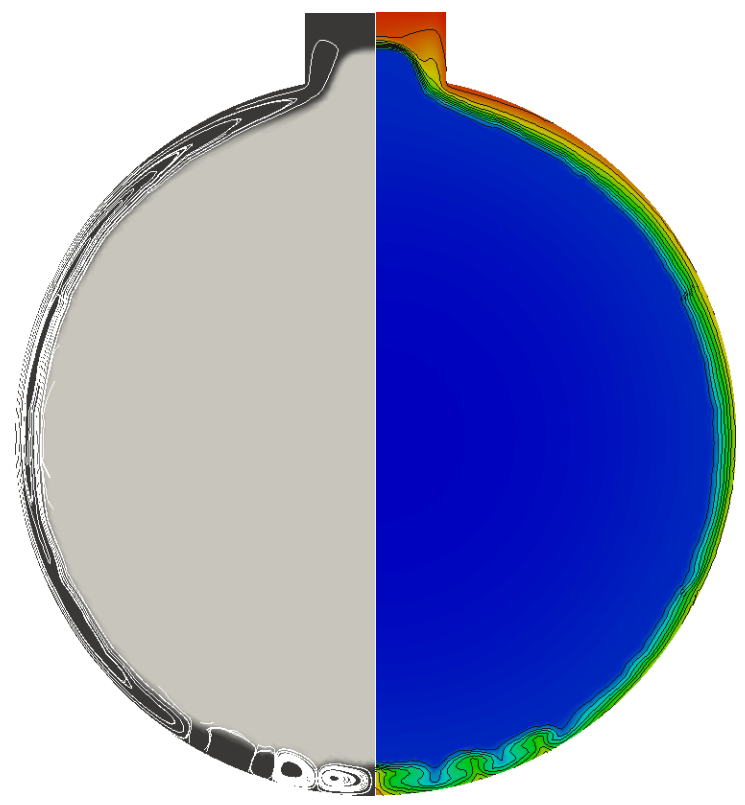

(c) Mesh: 2D-wo-9.3E3. Case C. Variable properties.

$$
\begin{array}{lr}
T(K) \\
\quad 308 \quad 312 \\
& 313.15
\end{array}
$$

$304 \quad 308,312$
313.15

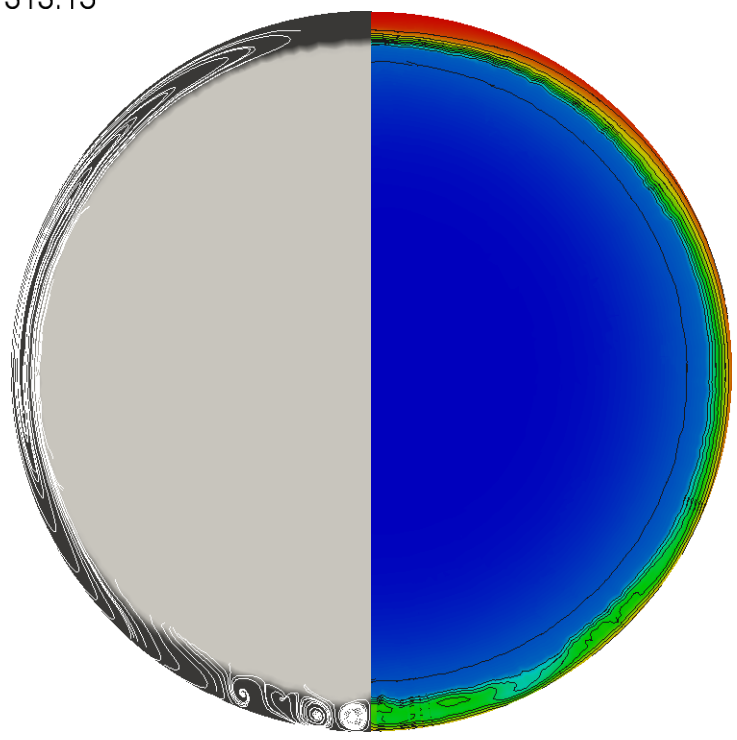

(b) Mesh: 3D-NO-1.4E6. Case A. Constant properties.

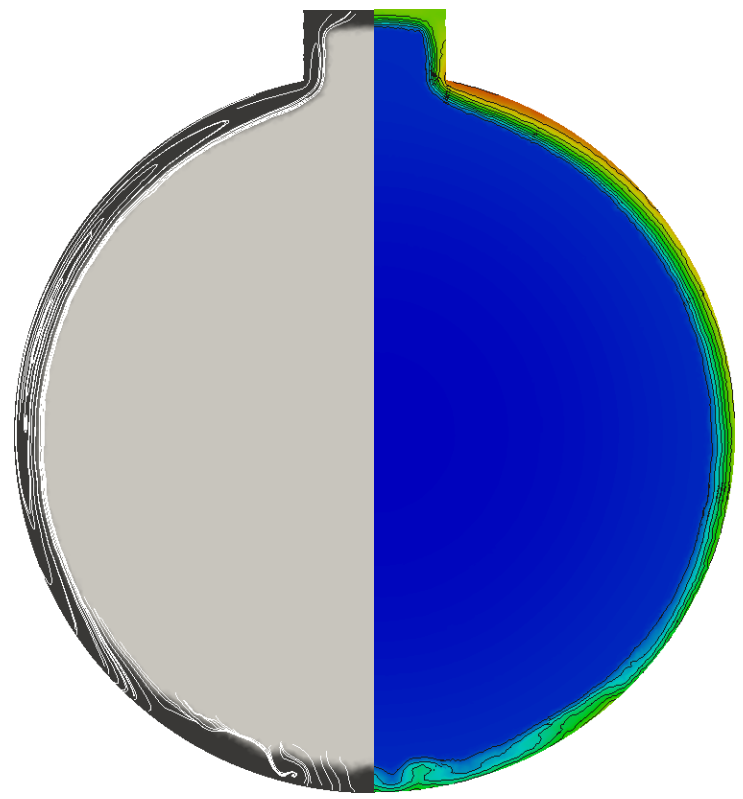

(d) Mesh: 3D-wo-5E6. Case C. Variable properties.

Figure 10: Vertical cross view of numerical results for $10 \mathrm{~min}$ of simulation ( $t \sim 22 \mathrm{~min}$ in the experiment). The left half of each picture shows a map of liquid fraction (light: solid; dark: liquid) with the flow streamlines, while the right half shows temperature map and contours. The density of the $3 \mathrm{D}$ mesh slices and $2 \mathrm{D}$ meshes shown are similar. 


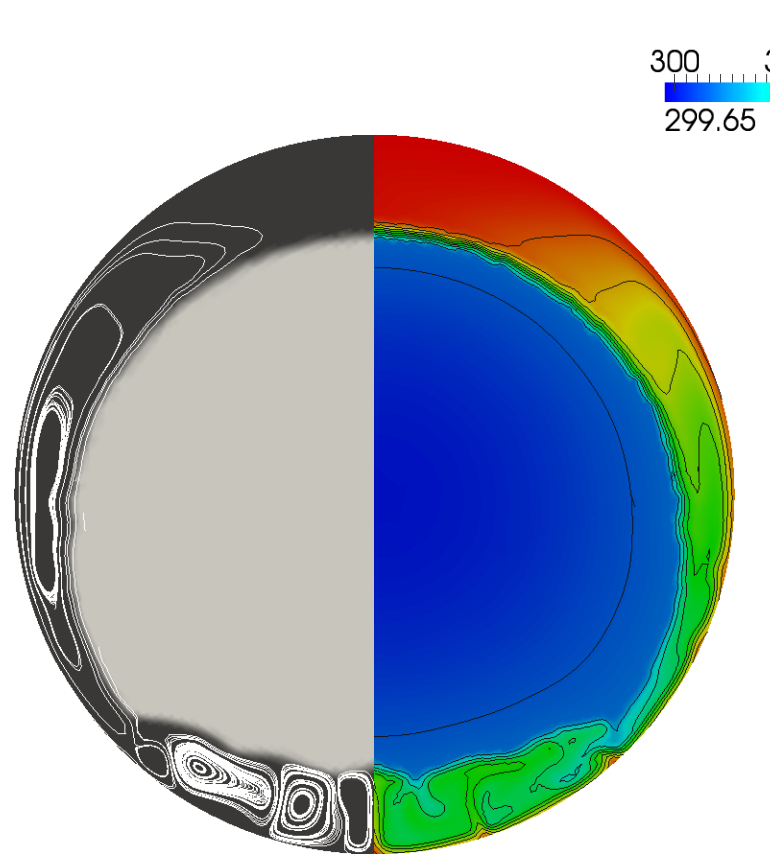

(a) Mesh: 2D-NO-7.7E3. Case A. Constant properties.

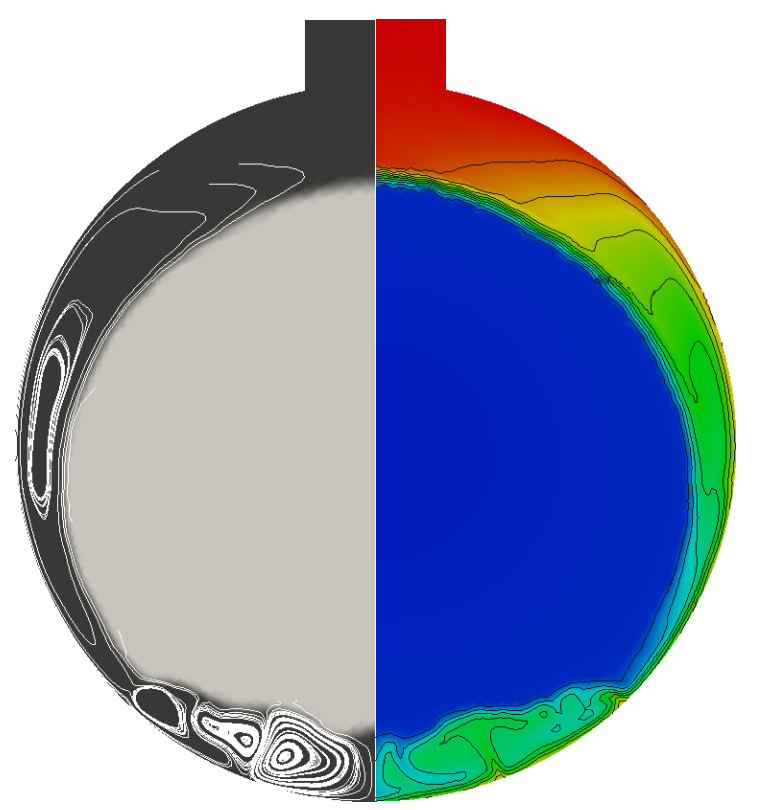

(c) Mesh: 2D-wo-9.3E3. Case C. Variable properties.

\section{$T(K)$

$308 \quad 312$

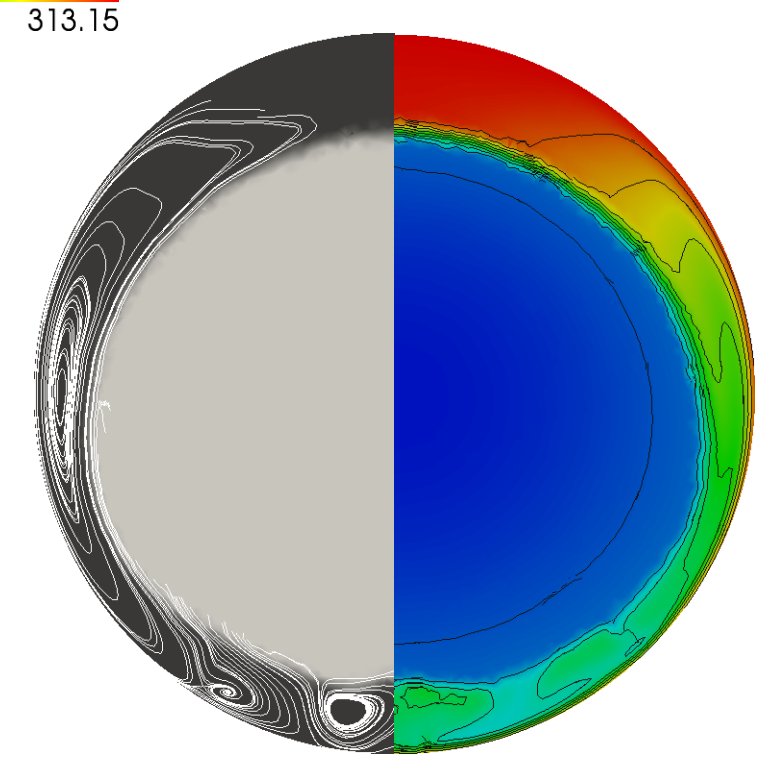

(b) Mesh: 3D-NO-1.4E6. Case A. Constant properties.

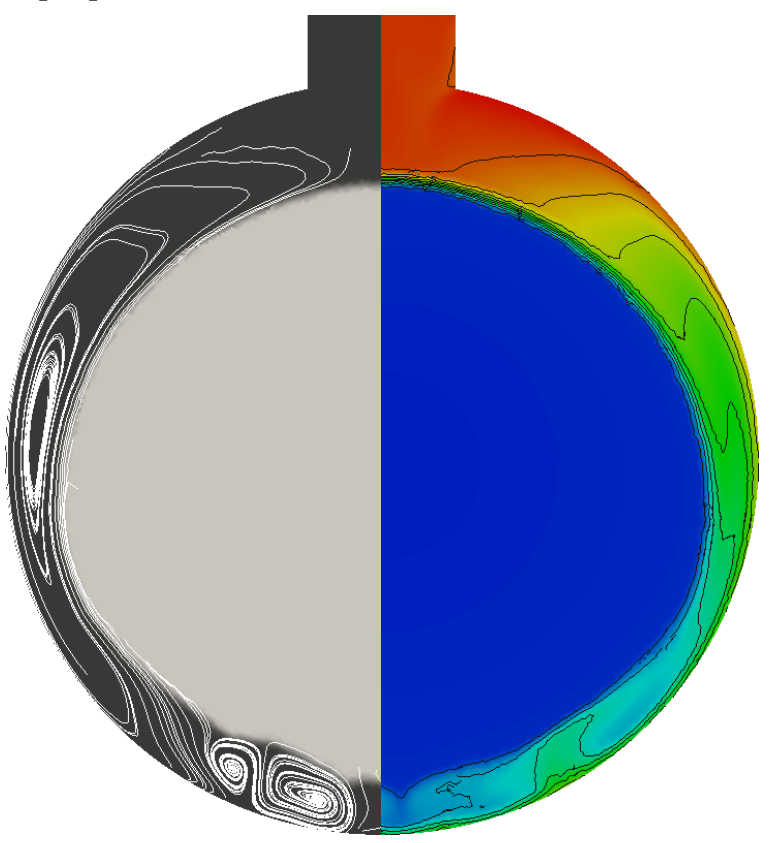

(d) Mesh: 3D-wo-5E6. Case C. Variable properties.

Figure 11: Vertical cross view of numerical results for $t_{\text {exp }} \sim 47 \mathrm{~min}$. The left half of each picture shows a map of liquid fraction (light: solid; dark: liquid) with the flow streamlines, while the right half shows temperature map and contours. The density of the 3D mesh slices and 2D meshes shown are similar. zones of higher and lower temperatures can be observed. This effect is present at the bottom of 674 the capsule but not at the top, where a thermal stratification is seen (nearly horizontally uniform 


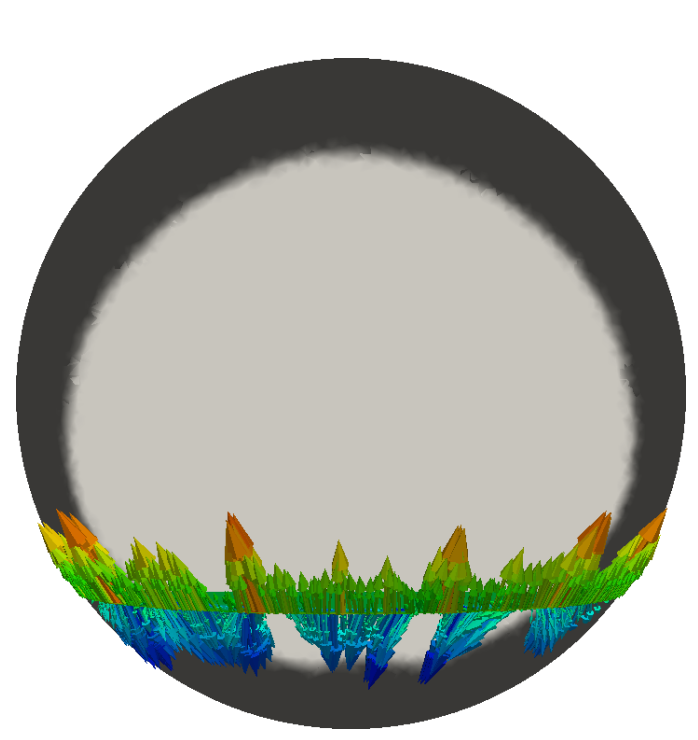

(a) Front view

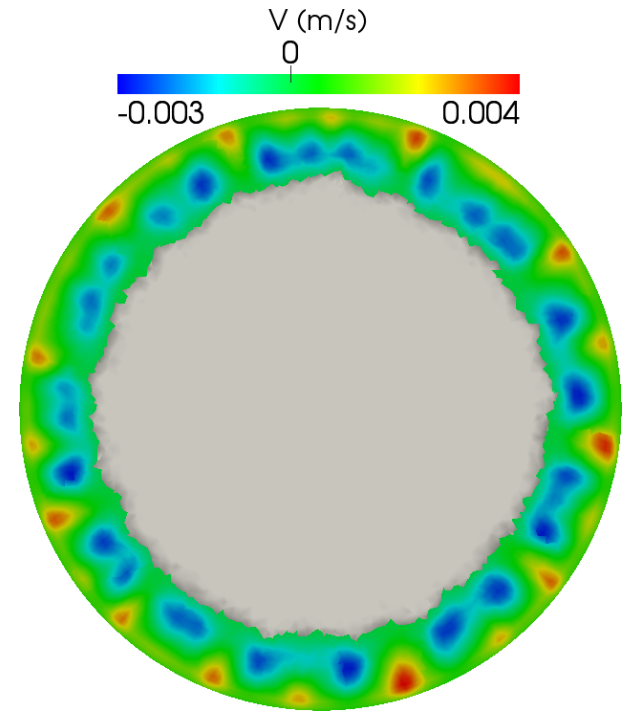

(b) Top view

Figure 12: Different views of slice cuts run with mesh 3D-NO-1.4E6 at $t_{e x p} \sim 47$ minutes (case A, const. props.). The horizontal cut is at $3 \mathrm{~cm}$ below the center of the sphere. Velocity vectors are shown in the front view, while a vertical velocity map is depicted in the top view of the horizontal cut. The greyscale indicates the phase (dark: liquid; light: solid), while the colors indicate the magnitude of the vertical component of the velocity.

temperature distribution) and the velocities are lower. In this figure it can also be noted that in case A the shell is hotter than in case $\mathrm{C}$, due to the higher heat transfer rate assumed for the former.

A consequence of the "artificial" trapping of the eddies in the 2D cases is that the lower part melts faster in these than in the 3D cases. This can be observed, not only by comparing the shapes of the interface, but also the evolution of the temperature at different axial positions, as shown in figs. 14-15. In the experimental results of Tan et al. [16], the thermocouple located at point $\mathrm{G}(y=2.5 \mathrm{~cm}$ above the center) detects that melting there starts before than at point B ( $y=3.75 \mathrm{~cm}$ below the center). In all 2D numerical results reported in this work, as well as in those published by [16] (numerical, 2D), point B starts melting long before point G. However, in 3D cases, melting of point B is much delayed, resulting in a better qualitative agreement with the experimental data.

Regarding the evolution of global liquid fraction, it is not clear if 3D simulations result in different melting rates. Results using the SYMMPRES scheme (Figs. 7c and 7f) seem to indicate that 3D simulations present slightly higher melting rates, but the discrepancies with $2 \mathrm{D}$ results are so small that could be due to differences in the used meshes. 


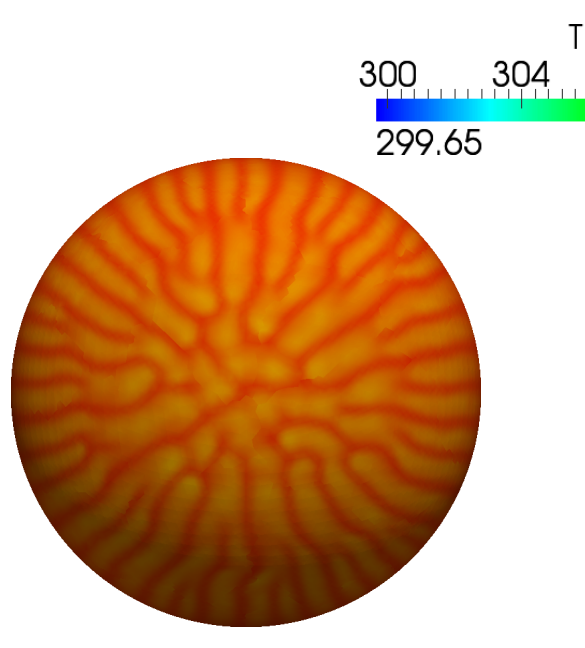

(a) Case A, const. props.; $t_{\exp } \sim 22$ min. Mesh: 3D-NO-1.4E6.

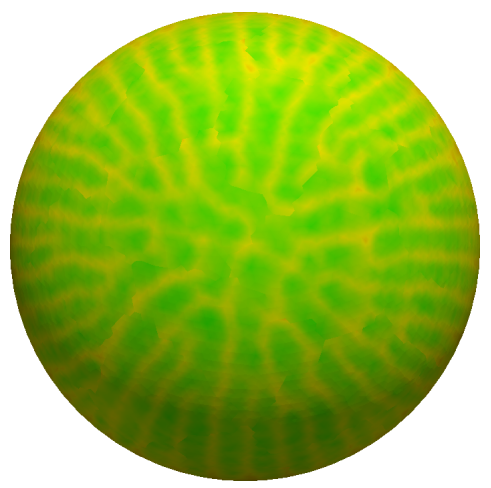

(c) Case C, var. props.; texp $\sim 22$ min. Mesh: 3D-WO-1.6E6.
$\mathrm{T}(\mathrm{K})$ $308 \quad 312$

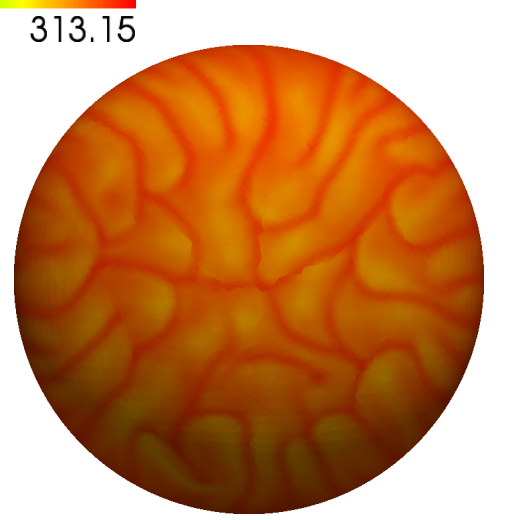

(b) Case A, const. props.; $\mathrm{t}_{\exp } \sim 32$ min. Mesh: 3D-NO-1.4E6.

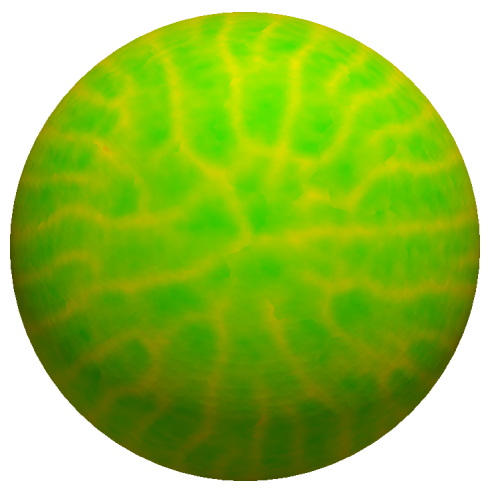

(d) Case C, var. props.; texp $\sim 32$ min. Mesh: 3D-Wo-1.6E6.

Figure 13: Bottom view of temperature maps in the shell, for 3D simulations.

\subsection{Agreement between experimental and numerical results}

All the numerical results reproduce very well the qualitative behavior observed in the experiment. Furthermore, a very good accordance in the evolution of the melting rate is obtained for the numerical results with both $2 \mathrm{D}$ and $3 \mathrm{D}$ meshes using the SYMMPRES scheme. However, the quantitative agreement with the local temperature measurements is not so good.

Figures 14-16 show higher temperature oscillations at locations below the center of the sphere for the numerical results, than those observed in the experimental data. This could be indicating that velocities higher than those occurring in the experiment might be being predicted in the simulations. This is specially true for 2D cases (Figs. 14a, 15a and 16a), where the high velocity flows generated at the bottom cannot escape to the top of the capsule, causing higher thermal oscillations in this zone. Furthermore, it is believed that the onset of the phase change in the experiment is produced at a lower temperature than that found in the literature $\left(28.18^{\circ} \mathrm{C}\right)$, and may be occurring in a temperature range 


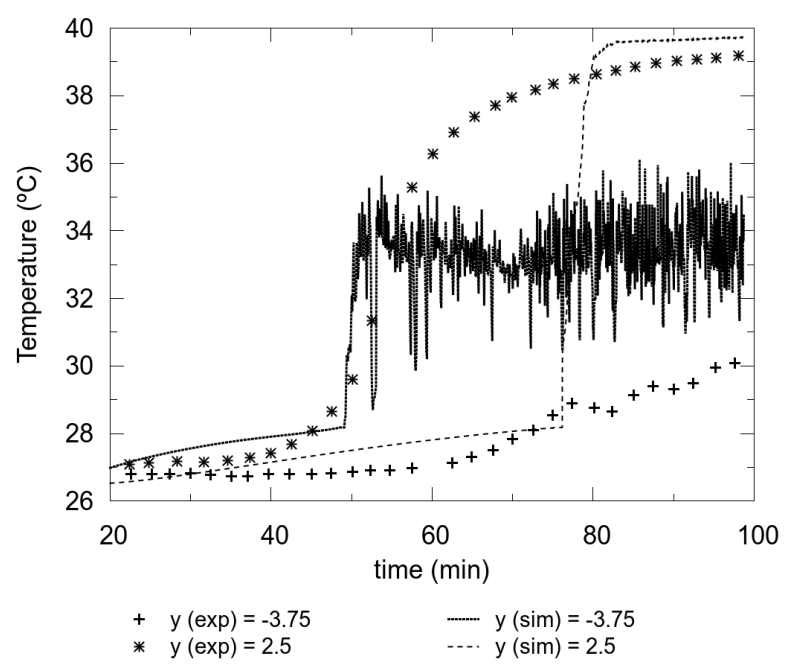

(a) Mesh: 2D-NO-31E3

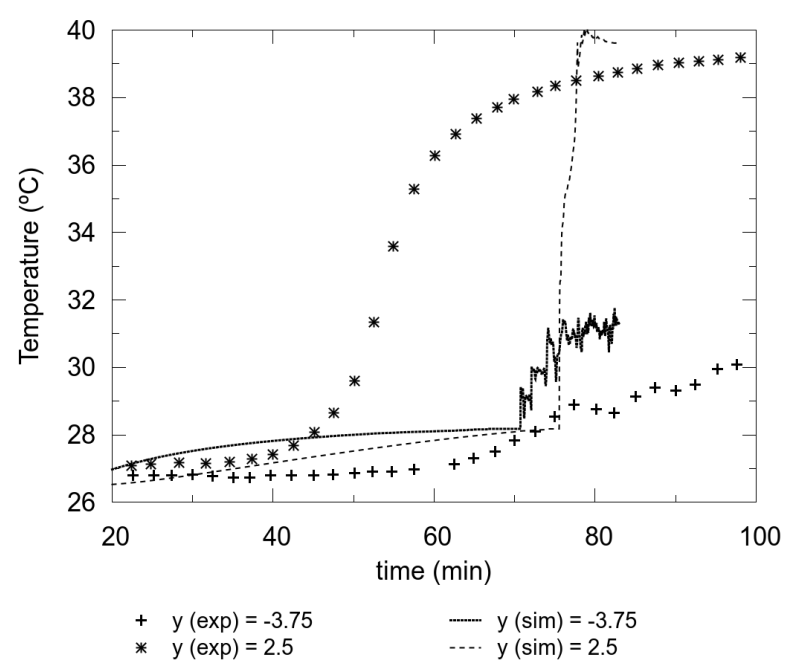

(b) Mesh: 3D-NO-1.4E6

Figure 14: Evolution of $\mathrm{T}$ vs. time, for numerical and experimental results (extracted from [16]), at two different locations of the vertical axis (indicated in $\mathrm{cm}$, with $y=0$ being the center of the capsule). Case A, using constant properties.

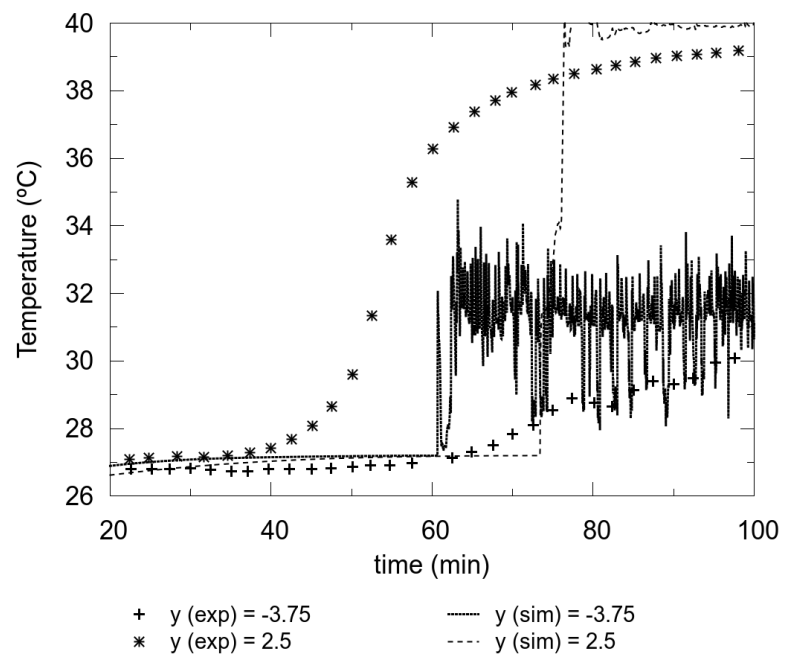

(a) Mesh: 2D-Wo-9.3E3

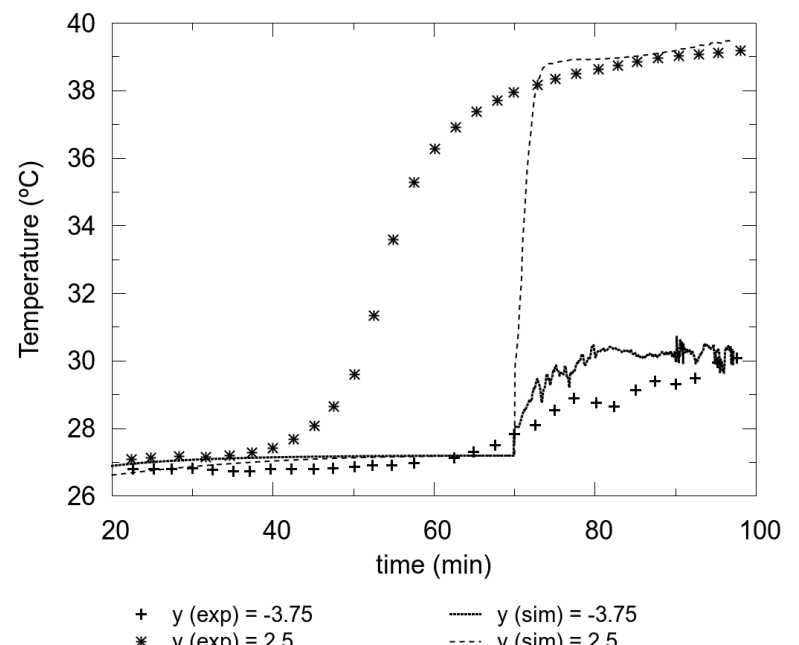

(b) Mesh: 3D-wo-1.6E6

Figure 15: Evolution of $\mathrm{T}$ vs. time, for numerical and experimental results (extracted from [16]), at two different locations of the vertical axis (indicated in $\mathrm{cm}$, with $y=0$ being the center of the capsule). Case $\mathrm{C}$, using variable properties.

of around $27-29^{\circ} \mathrm{C}$. This would explain that the experimental temperature curves stay nearly horizontal around $27^{\circ} \mathrm{C}$ and rise more "smoothly" than in the numerical results. By adopting a melting point of $27.2^{\circ} \mathrm{C}$ (case $\mathrm{C}$ ), the temperature evolution of the solid phase is better predicted, but the increase after melting is still much higher than in the experiment. In $3 \mathrm{D}$ results of case $\mathrm{C}$ with the densest mesh 


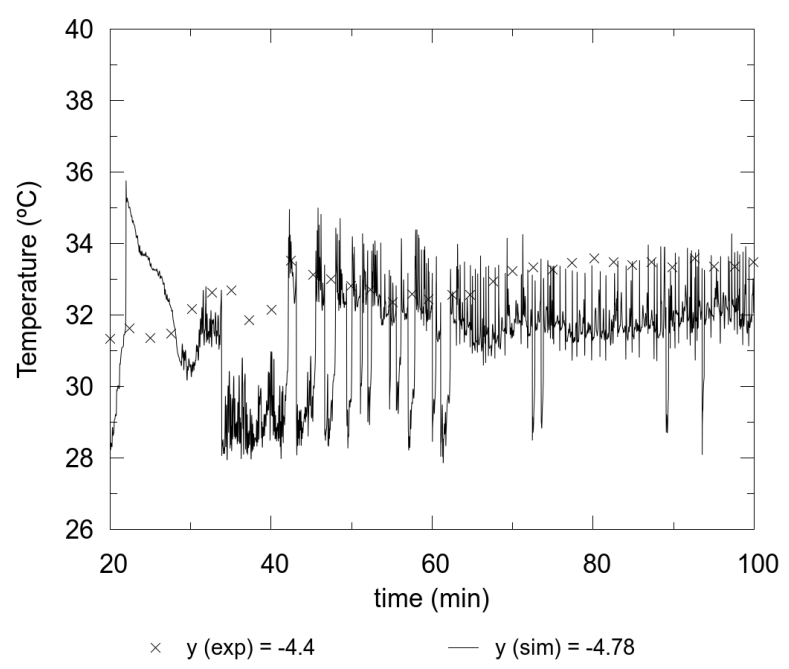

(a) Mesh: 2D-Wo-9.3E3

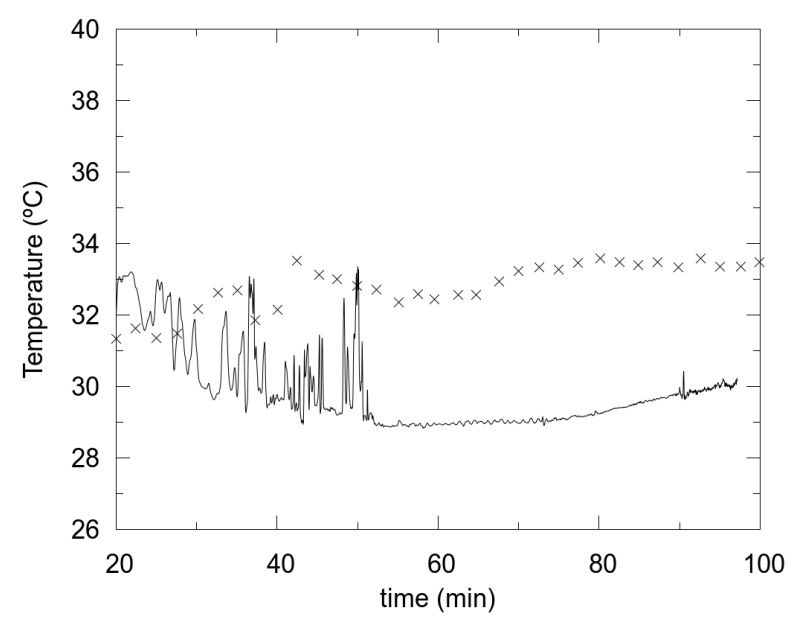

$\times \quad y(\exp )=-4.4 \quad-y(\operatorname{sim})=-4.78$

(b) Mesh: 3D-wo-1.6E6

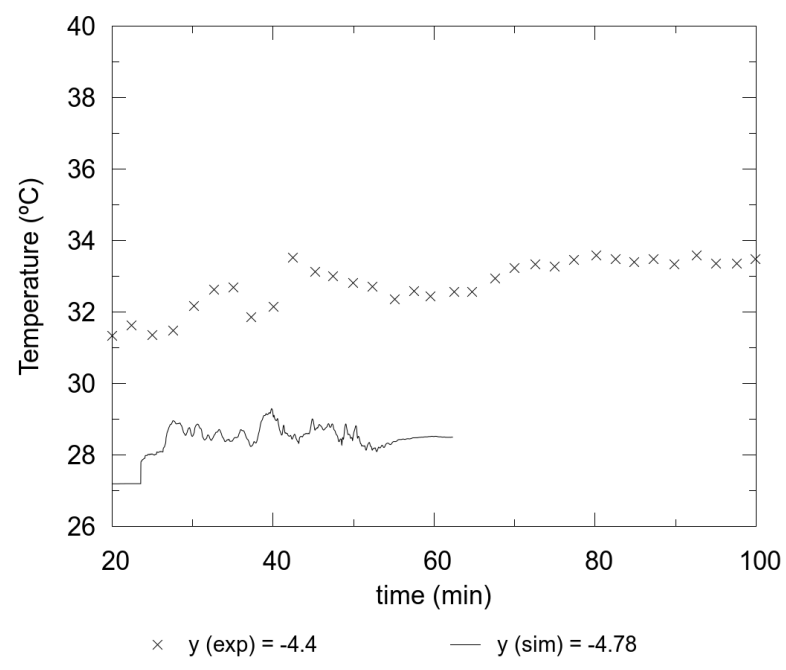

(c) Mesh: 3D-WO-5E6

Figure 16: Evolution of $\mathrm{T}$ vs. time, for numerical and experimental results (extracted from [16]), at a location close to the bottom, in the vertical axis (indicated in $\mathrm{cm}$, with $y=0$ being the center of the capsule). Case $\mathrm{C}$, using variable properties.

(3D-WO-5E6, Fig. 16c), the amplitude of the temperature oscillations at position $\mathrm{A}$ is very similar to the observed in the experimental data, and lower than the obtained with the coarser 3D mesh (3D-Wo-1.6E6, Fig. 16b). However, the predicted mean value is somewhat lower in both cases, which may be another indication of the presence of solid phase at a temperature higher than the assumed melting point, in the experiment.

In figures 17 and 18, the interface shapes at different instants for both experimental and numerical results are presented. A very good agreement with the experiment is observed in general for the 3D simulations, specially for those using variable properties; while the effect of eddy trapping (mentioned 


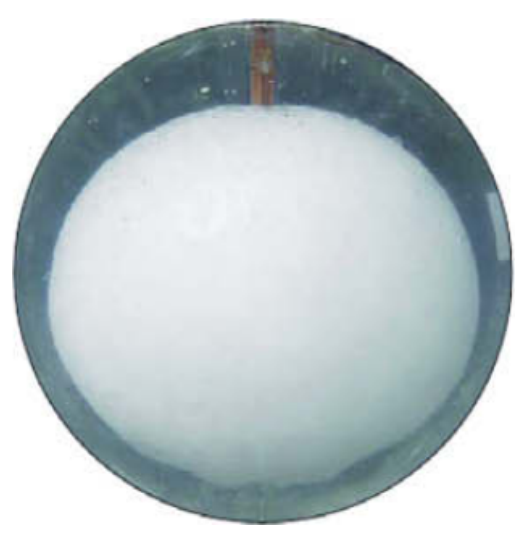

(a) Experiment ( $t=40 \mathrm{~min})$.

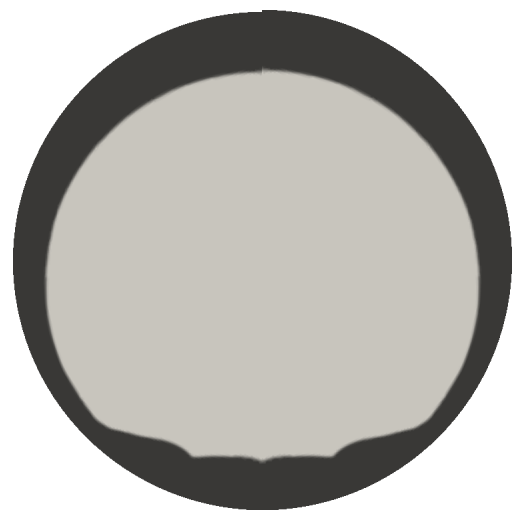

(d) Numerical (t $\sim 40 \quad \min )$.

Mesh: 2D-NO-32E3.

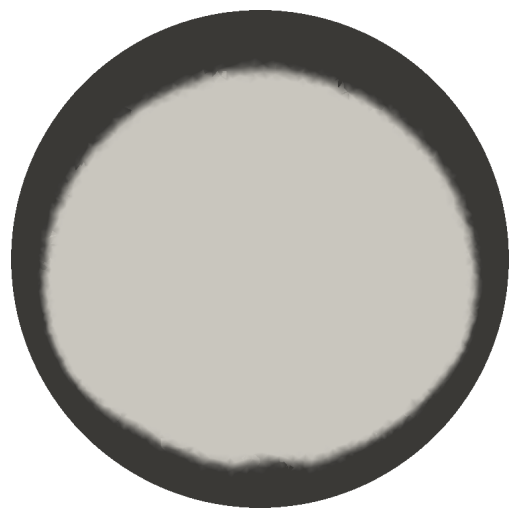

(g) Numerical (t 40 min). Mesh: 3D-NO-1.4E6.

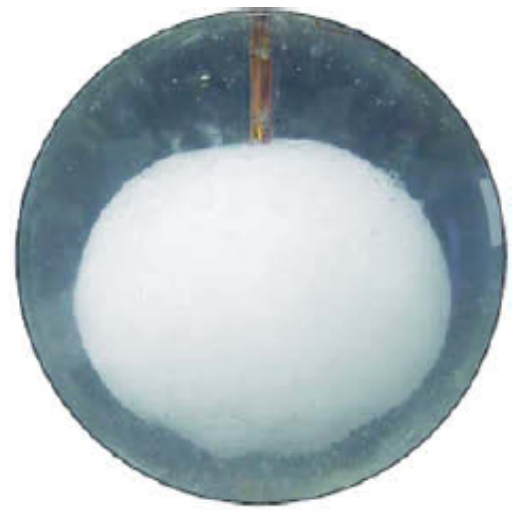

(b) Experiment ( $\mathrm{t}=60 \mathrm{~min})$.

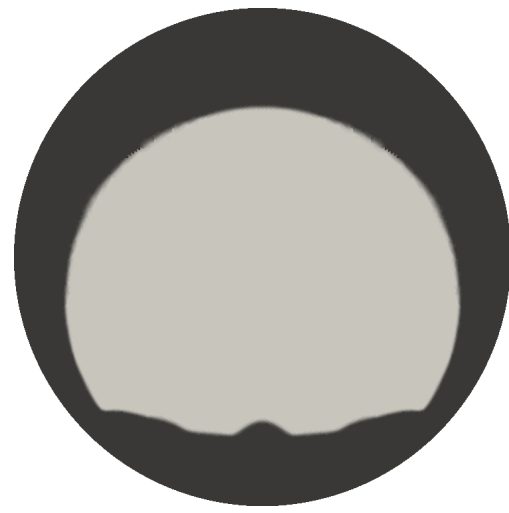

(e) Numerical (t $\sim 60$ min). Mesh: 2D-NO-32E3.

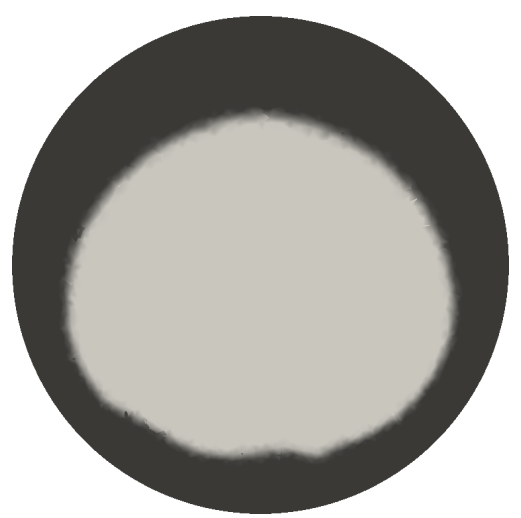

(h) Numerical $\quad(\mathrm{t} \sim 60 \quad \mathrm{~min})$. Mesh: 3D-NO-1.4E6.

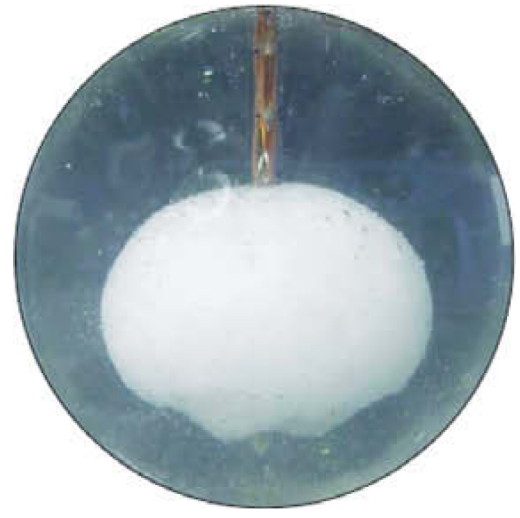

(c) Experiment $(\mathrm{t}=80 \mathrm{~min})$.

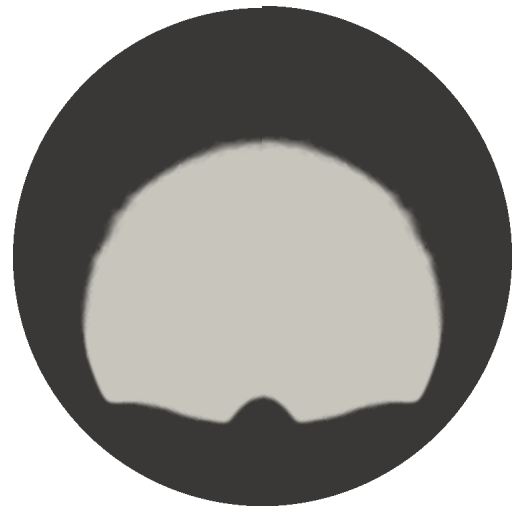

(f) Numerical (t $\sim 80 \mathrm{~min})$. Mesh: 2D-NO-32E3.

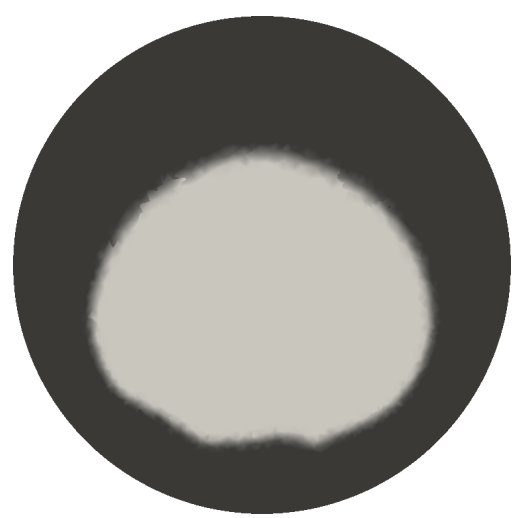

(i) Numerical (t 80 min). Mesh: 3D-NO-1.4E6.

Figure 17: Experimental photographs at $t_{\text {exp }}=40,60$ and 80 minutes (extracted from [16]) and our numerical results for case A, using constant properties, with 2D and 3D meshes. Liquid fraction map (dark: liquid; light: solid) is shown for the numerical results.

above) produce a higher disagreement in $2 \mathrm{D}$ cases.

Regarding the thermal boundary condition, it is difficult to draw clear conclusions about which of both values of $\alpha_{e q}$ is more accurate. Observing global liquid fraction curves for case $\mathrm{A}\left(\alpha_{e q}=\right.$ 


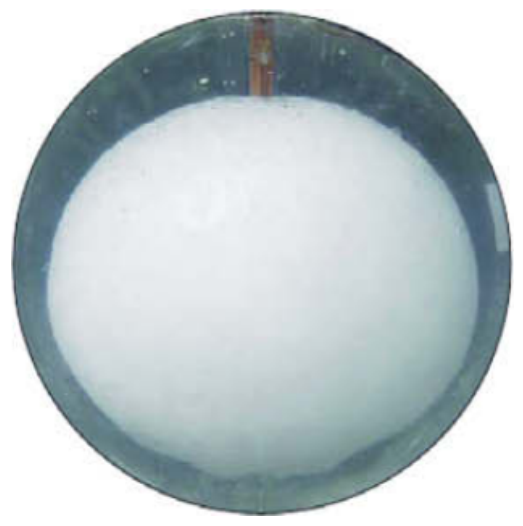

(a) Experiment ( $t=40 \mathrm{~min})$.

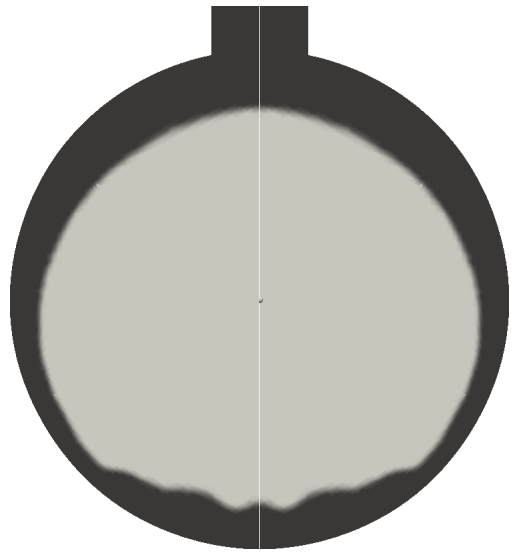

(d) Numerical (t 40 $\min )$. Mesh: 2D-Wo-9.3E3.

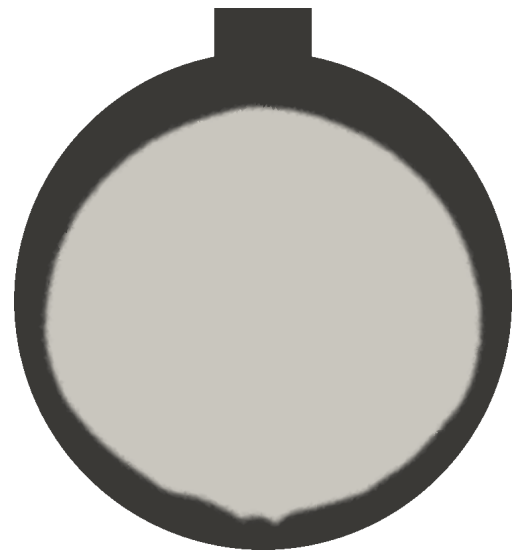

(g) Numerical (t 40 min). Mesh: 3D-WO-5E6.

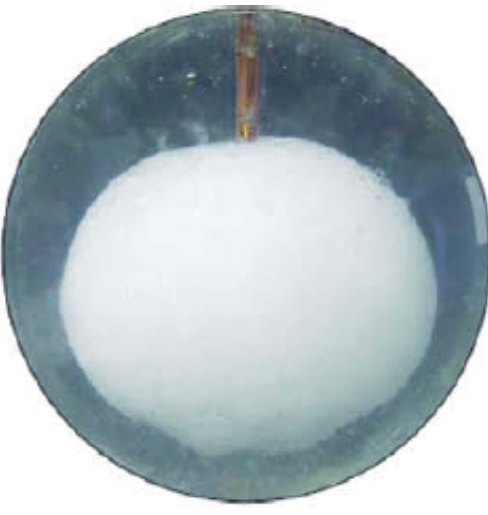

(b) Experiment ( $\mathrm{t}=60 \mathrm{~min})$.

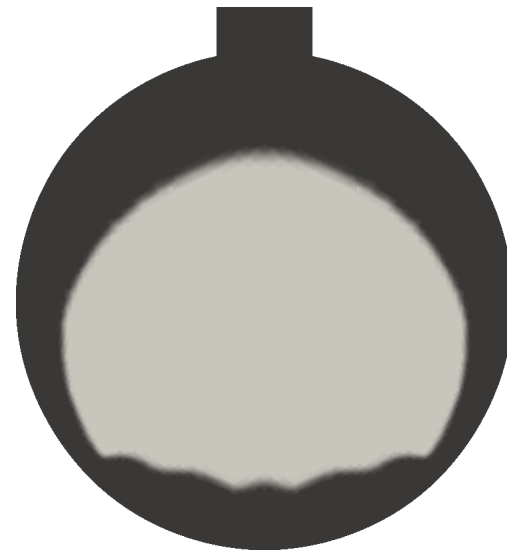

(e) Numerical (t $\sim 60$ min). Mesh: 2D-WO-9.3E3.

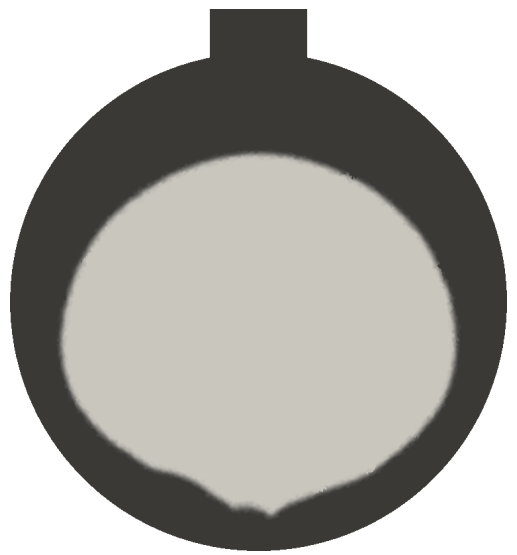

(h) Numerical (t $\sim 60 \quad \mathrm{~min})$. Mesh: 3D-WO-5E6.

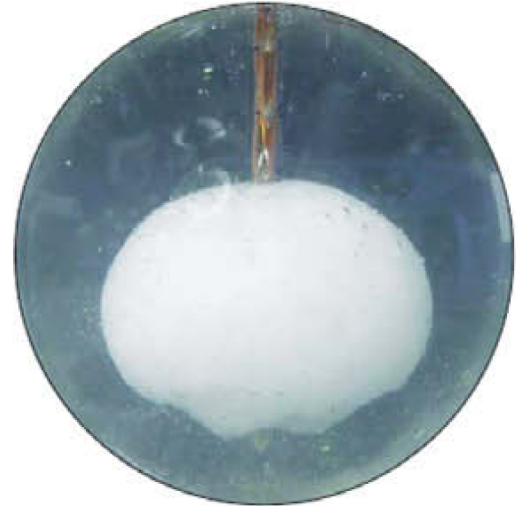

(c) Experiment $(\mathrm{t}=80 \mathrm{~min})$.

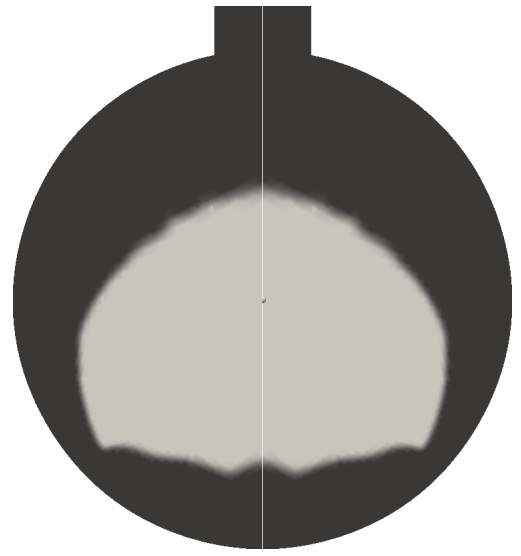

(f) Numerical (t 80 min). Mesh: 2D-WO-9.3E3.

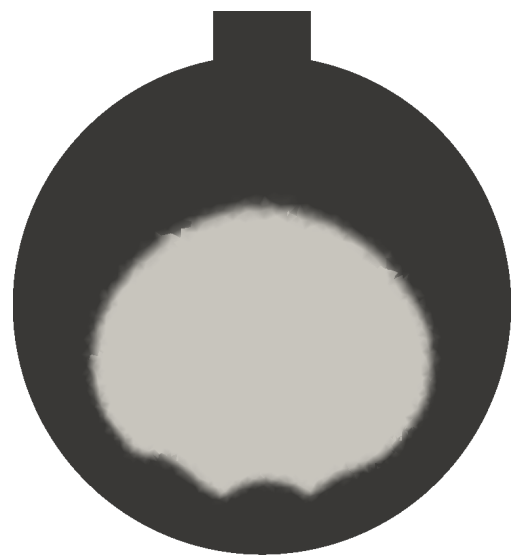

(i) Numerical (t $\sim 80 \min )$. Mesh: 3D-WO-1.6E6.

Figure 18: Experimental photographs at $t_{\text {exp }}=40,60$ and 80 minutes (extracted from [16]) and our numerical results for case $\mathrm{C}$, using variable properties, with 2D and 3D meshes. Liquid fraction map (dark: liquid; light: solid) is shown for the numerical results. 
$796 \mathrm{~W} / \mathrm{m}^{2} \mathrm{~K}$ and $T_{s l}=28.2^{\circ} \mathrm{C}$ ) with both constant and variable properties, and comparing the results obtained with 2D meshes of similar amount of cells (2D-NO-7.7E3 and 2D-WO-9.3E3), it is apparent that the melting rate is overpredicted with respect to the experimental data, specially if the variation of the thermo-physical properties is taken into account. However, in simulations using denser 2D meshes, it is observed that for both constant and variable properties the melting rate obtained is very close to that of the experiment (Figs. $7 \mathrm{c}$ and $7 \mathrm{~d}$ ). On the other hand, for a melting point of $28.2^{\circ} \mathrm{C}$, the value of $\alpha_{e q}=237 \mathrm{~W} / \mathrm{m}^{2} \mathrm{~K}$ (case B) underpredict the real melting rate (see 7e).

For 3D simulations of case $\mathrm{C}$ (with the lower melting point, variable properties and $\alpha_{e q}=$ $\left.237 \mathrm{~W} / \mathrm{m}^{2} \mathrm{~K}\right)$, the predicted melting rate is still somewhat higher than experimental, even for the densest mesh (3D-WO-5E6). Here, the higher difference between the water bath temperature and the melting point is expected to result in stronger convection than in case A, and thus, in higher melting rates for the same value of $\alpha_{e q}$. This could explain the very good results obtained with the lower $\alpha_{e q}$.

In the belief that the material used in the experiment undergoes a phase change at a temperature range, it is possible that the solid phase is still present at temperatures higher than $28^{\circ} \mathrm{C}$. Therefore, it is possible that the convection is not as strong as that resulting from the phase transition at a fixed melting point of $27.2^{\circ} \mathrm{C}$, and thus, the higher value of $\alpha_{e q}$ is believed to be more accurate. Nevertheless, when comparing the melting rates of cases $\mathrm{A}$ and $\mathrm{B}$ with the same mesh 2D-Wo-9.3E3, i.e. $0.9 \% / \mathrm{min}$ and $0.78 \% / \mathrm{min}$, a difference of around $13-15 \%$ is obtained, which is not much, considering that $\alpha_{e q}$ for case A is three times higher than for case B. Furthermore, if the sensitivity of the melting rate with the external convection coefficient (between the water bath and the external surface of the glass shell) is considered, it can be said that it is significantly low in the range considered, since the difference in this value for cases $\mathrm{A}$ and $\mathrm{B}$ is of around 10 times $\left(\sim 3000 \mathrm{~W} / \mathrm{m}^{2} \mathrm{~K}\right.$ vs. $\sim 300 \mathrm{~W} / \mathrm{m}^{2} \mathrm{~K}$, respectively).

A further aspect that could be influencing the melting rate is the presence of the hollow tube (apparently metallic), which could be increasing the heat flux into the solid phase and increasing the melting rate near the axis. Slight deformation of the interface near the rod (at the top) seems to be appreciated in the experimental figures, which could indicate that this phenomenon might be occurring, although it does not seem very strong. Furthermore, the hollow tube displaces some amount of n-Octadecane, which could also result in a faster total melting rate.

In summary, with the use of an accurate melting temperature range, as well as more accurate boundary conditions, three-dimensional numerical simulations would most probably be even closer to experimental results. Regarding thermo-physical properties, of special importance in this case is to have a more accurate value of the solid phase density, since it affects the total amount of latent energy initially contained in the capsule. It also affects the expansion in the melting, although this 
phenomenon has not been seen to influence the melting rate significantly in this case.

\subsection{Computational cost}

The difference in computational cost between the different cases depend on several aspects, such as if it is $2 \mathrm{D}$ or $3 \mathrm{D}$, the number of processors used and the refinement of the mesh. Since the meshes used are unstructured, using two meshes of around the same number of cells but with different local refinement the time steps of the simulations result different, and therefore, the computational cost is also different.

Since the cases with 2D meshes were mostly run on a desktop computer (using 2 processors of the 4 available), the added difficulty is that at the same time the computer could be being used for other tasks and the resources were shared, and therefore, a value of computational time cannot be indicated very accurately.

However, some general information is here presented, in order to approximately indicate the cpu time needed for some of the simulations and to have an idea about the differences in computational costs of running 2D and 3D simulations usign constant and variable properties.

A significant difference has been observed between the computational costs of cases using constant and variable thermo-physical properties, being closely related to the number of iterations of the pressure solver needed for each time step. For example, for case A with constant properties and mesh 2D-NO-7.7E3, using $2 \mathrm{cpu}$ cores, the number of solver iterations per time step was around 20, while for the same case but using variable properties and mesh 2D-WO-9.3E3 (also divided in 2 cores) this value was around 200. This difference is probably due to the extra source term resulting from the density variations in the pressure system of equations [compare equations (11) and (15)] which makes the problem harder to solve for the linear solver. This fact, along with the smaller time steps of case with mesh 2D-WO-9.3E3 (around half), due to the higher mesh refinement (compare the values of $r_{e q, \min }$ in Table 1), resulted in ratios of cpu/physical time of around 5-10 for the case with mesh 2D-NO-7.7E3 and 50-150 for the one with mesh 2D-WO-9.3E3.

In $3 \mathrm{D}$ simulations, for case $\mathrm{A}$ with constant properties and mesh 3D-NO-1.4E3, using 64 cores, the number of solver iterations per time step was around 15-45, while for the same case with variable properties and mesh 3D-WO-1.6E3 (also in 64 cores), this number was around 50-300. The difference in the time steps between these cases was not so significant as between $2 \mathrm{D}$ cases $(\sim 10 \%$ bigger in the latter). Hence, the cpu/physical time ratio was around 50-350 in the former and around 200-900 in the latter.

In summary, for these cases, it could be said that the computational time needed for running $3 \mathrm{D}$ cases is in the order of 10 times higher than for $2 \mathrm{D}$ cases; while those with variable properties 
need around 6 times higher computational time (for the same time steps) than those with constant properties.

\section{Conclusions}

A fixed-grid enthalpy model for unstructured grids using explicit time integration where the thermo-physical properties are variable and dependent on the temperature, has been developed and implemented in a numerical code. The formulation and algorithms for determining the temperature and liquid fraction (or porosity) from the total enthalpy, for phase change occurring at a fixed temperature or in a temperature range, have been described. Modifications of the model presented in [1], regarding different aspects such as the treatment of the conservation equations taking into account the thermal expansion/contraction, the approach for decoupling the pressure of solid and liquid-containing domains and the determination of the source term coefficient introduced by the porous medium approach, have been detailed. Furthermore, different possibilities for the discretization of the energy equation have been discussed.

With the intention of accurately simulating the case of melting of n-Octadecane inside a spherical capsule taking into consideration both accurate constant properties as well as their variation with the temperature, a thorough search for thermo-physical properties has been performed. The correlations used have been presented, indicating their origin and accuracy (either quantitatively or qualitatively). Furthermore, it is indicated how in cases where the density differences between liquid and solid phases is not accounted for, the latent heat should be modified in order to obtain the same amount of total enthalpy inside the same control volume.

Several two and three-dimensional simulations of the case study have been performed. The discretization of the convective scheme of the energy equation has been observed to affect considerably the results, for which the symmetry preserving scheme seem to give more accurate results than the first-order UPWIND scheme. From the comparison between 2D and 3D results, significant differences have been encountered in the flow patterns developed, originated in the unstable thermal zone in the bottom of the capsule. These different flow structures result in different melting rates in the bottom zone near the axis of symmetry, although the evolution of global liquid fraction does not appear to be significantly different between $2 \mathrm{D}$ and $3 \mathrm{D}$ simulations. Comparison against experimental results allow to state that the agreement obtained with the 3D simulations is higher than with those assuming axi-symmetry (2D).

Regarding the thermo-physical properties used, it has been shown that when using the liquid density for both phases, scaling the latent heat with the density ratio (solid/liquid) compensate for the 
error in the total energy per unit volume needed for producing the melting, and more accurate results of the evolution of global liquid fraction are obtained. Furthermore, it is observed that accounting for the full variation of the properties with the temperature results in slightly higher convective fluxes and overall faster melting, which has been seen to be mostly due to the change in the thermal conductivity.

On the other hand, it is apparent from the experimental curves of temperature evolution at some locations, that the material undergoes a phase-change in a range of temperatures instead of at a fixed melting point, which can be a cause of discrepancy between experimental and numerical results.

Another source of discrepancy is the thermal boundary condition in the capsule shell, which in this work has been treated as an equivalent heat transfer coefficient between the thermal bath and the internal surface of the shell and a fixed temperature for the thermal bath. Two different values for this coefficient have been tested. The highest of both resulted in melting rates closer to the experiment in the cases where a melting point of $28.2^{\circ} \mathrm{C}$ was assumed, while the lowest resulted in better agreement for the cases with a melting point of $27.2^{\circ} \mathrm{C}$. Since it is apparent that the solid phase may still be present at temperatures higher than $28.2^{\circ} \mathrm{C}$, it is believed that simulations considering the phase change temperature range, may be closer to the experimental results if a heat transfer coefficient closer to the highest of both is used. Nevertheless, it is observed that the difference in the melting rates obtained with one or another value for the same mesh, are much less significant than the difference between them.

Overall, a good agreement between experiment and numerical results is observed, specially for $3 \mathrm{D}$ simulations taking into account the variation of thermo-physical properties with the temperature. Results obtained in the present work are closer to the experimental results than those presented by other authors $[16,17]$.

\section{Acknowledgements}

This work has been financially supported by the Ministerio de Economía y Competitividad, Secretaría de Estado de Investigación, Desarrollo e Innovación, Spain (ENE-2011-28699), by Termo Fluids S.L., by the EIT via the KIC InnoEnergy TESCONSOL project (ref. 20_2011_IP16), by the Secretaria d'Universitats i Recerca (SUR) del Departament d'Economia i Coneixement (ECO) de la Generalitat de Catalunya and by the European Social Fund.

\section{References}

[1] P. Galione, O. Lehmkuhl, J. Rigola, and A. Oliva, "Fixed-grid modeling of solid-liquid phase change in unstructured meshes using explicit time schemes," Numer. Heat Tr. B-Fund., vol. 65, 
pp. 27-52, 2014 .

[2] H. Michels and R. Pitz-Paal, "Cascaded latent heat storage for parabolic trough solar power plants," Sol. Energy, vol. 81, pp. 829-837, 2007.

[3] W. Steinmann and R. Tamme, "Latent heat storage for solar steam systems," J. Sol. Energ.-T. ASME, vol. 130, pp. 011004-1-011004-5, 2008.

[4] M. Liu, W. Saman, and F. Bruno, "Review on storage materials and thermal performance enhancement techniques for high temperature phase change thermal storage systems," Renew. Sust. Energ. Rev., vol. 16, pp. 2118-2132, 2012.

[5] S. Jegadheeswaran and S. D. Pohekar, "Performance enhancement in latent heat thermal storage system: A review," Renew. Sust. Energ. Rev., vol. 13, pp. 2225-2244, 2009.

[6] K. A. R. Ismail and R. Stuginsky Jr., "A parametric study on possible fixed bed models for pcm and sensible heat storage," Appl. Therm. Eng., vol. 19, pp. 757-788, 1999.

[7] J. P. Bédécarrats, J. Castaing-Lasvignottes, F. Strub, and J. P. Dumas, "Study of a phase change energy storage using spherical capsules. Part II: Numerical modelling," Energ. Convers. Manage., vol. 50, pp. 2537-2546, 2009.

[8] S. M. Flueckiger and S. V. Garimella, "Latent heat augmentation of thermocline energy storage for concentrating solar power -A system-level assessment," Appl. Energ., vol. 116, pp. 278-287, 2014 .

[9] C.-J. Ho and R. Viskanta, "Heat transfer during melting from an isothermal vertical wall," $J$. Heat Trans.-T. ASME, vol. 106, pp. 12-19, 1984.

[10] F. Wolff and R. Viskanta, "Melting of a pure metal from a vertical wall," Exp. Heat Transfer, vol. 1, pp. 17-30, 1987.

[11] R. Viswanath and Y. Jaluria, "A comparison of different solution methodologies for melting and solidification problems in enclosures," Numer. Heat Tr. B-Fund., vol. 24, pp. 77-105, 1993.

[12] V. Voller and C. Prakash, "A fixed grid numerical modelling methodology for convection-diffusion mushy region phase change problems," Int. J. Heat Mass Tran., vol. 30, pp. 1709-1719, 1987.

[13] H. S. Udaykumar, R. Mittal, and W. Shyy, "Computation of solid-liquid phase fronts in the sharp interface limit on fixed grids," J. Comput. Phys., vol. 153, pp. 535-574, 1999. 
[14] A. D. Brent, V. R. Voller, and K. J. Reid, "Enthalpy-porosity technique for modelling convectiondiffusion phase change: Application to the melting of a pure metal," Numer. Heat Transfer, vol. 13, pp. 297-318, 1988.

[15] E. Assis, L. Katsman, G. Ziskind, and R. Letan, "Numerical and experimental study of melting in a spherical shell," Int. J. Heat Mass Tran., vol. 50, pp. 1790-1804, 2007.

[16] F. L. Tan, S. F. Hosseinizadeh, J. M. Khodadadi, and L. Fan, "Experimental and computational study of constrained melting of phase change materials (PCM) inside a spherical capsule," Int. J. Heat Mass Tran., vol. 52, pp. 3464-3472, 2009.

[17] W. Zhao, Elmozughi, A.F., A. Oztekin, and S. Neti, "Heat transfer analysis of encapsulated phase change material for thermal energy storage," Int. J. Heat Mass Tran., no. 63, pp. 323-335, 2013.

[18] A. Elmozughi, L. Solomon, A. Oztekin, and S. Neti, "Encapsulated phase change material for high temperature thermal energy storage - Heat transfer analysis," Int. J. Heat Mass Tran., vol. 78, pp. 1135-1144, 2014.

[19] D. A. Nield and A. Bejan, Convection in Porous Media, ch. 1. Springer, 3 ed., 2006.

[20] W. Rohsenow, J. Hartnett, and Y. Cho, Handbook of Heat Transfer, ch. 9. 1998.

[21] C. Hsu and P. Cheng, "Thermal dispersion in a porous medium," Int. J. Heat Mass Tran., vol. 33, pp. 1587-1597, 1990.

[22] P. Galione, Lehmkuhl, O., J. Rigola, A. Oliva, and R. I, "Numerical simulations of energy storage with encapsulated phase change materials. Special emphasis on solid-liquid phase change CFD modelling," in Proc. 12 ${ }^{\text {th }}$ Int. Conf. Energy Storage (INNOSTOCK), no. INNO-SP-56, 2012.

[23] O. Lehmkuhl, C. D. Pérez-Segarra, R. Borrell, M. Soria, and A. Oliva, "TERMOFLUIDS: A new Parallel unstructured CFD code for the simulation of turbulent industrial problems on low cost PC Cluster," Lect. Notes Comput. Sci. Eng., vol. 67, pp. 275-282, 2009.

[24] F. X. Trias and O. Lehmkuhl, "A self-adaptive strategy for the time integration of Navier-Stokes equations," Numer. Heat Tr. B-Fund., vol. 60, no. 2, pp. 116-134, 2011.

[25] O. Lehmkuhl, Numerical resolution of turbulent flows on complex geometries. PhD thesis, Universitat Politècnica de Catalunya, 2012.

[26] J. Ferziger and M. Perić, Computational Methods for Fluid Dynamics, ch. 7. Springer, 2002. 
[27] F. L. Tan, "Constrained and unconstrained melting inside a sphere," Int. Commun. Heat Mass, vol. 35 , pp. $466-475,2008$.

[28] http://www.ansys.com/Products/Other+Products/ANSYS+ICEM+CFD. ANSYS ICEM CFD.

[29] R. W. C. P. Verstappen and A. E. P. Veldman, "Symmetry-preserving discretization of turbulent flow," J. Eng. Math., vol. 187, no. 1, pp. 343-368, 2003.

[30] L. Jofre, O. Lehmkuhl, J. Ventosa, F. X. Trias, and A. Oliva, "Conservation properties of unstructured finite-volume mesh schemes for the Navier-Stokes equations," Numer. Heat Tr. B-Fund., vol. 65, no. 1, pp. 53-79, 2014.

[31] J. Messerly, G. Guthrie, S. Todd, and H. Finke, "Low-temperature thermal data for n-Pentane, n-Heptadecane and n-Octadecane," J. Chem. Eng. Data, vol. 12, pp. 338-346, 1967.

[32] M. Broadhurst, "An analysis of the solid phase behavior of the normal paraffins," J. Res. Nat. Bur. Stand., A: Phys. Chem., vol. 66, pp. 241-249, 1962.

[33] A. Briard, M. Bouroukba, Petitjean, D., N. Hubert, and M. Dirand, "Experimental enthalpy increments from the solid phases to the liquid phase of homologous n-alkane series (C18 to C38 and C41, C44, C46, C50, C60)," J. Chem. Eng. Data, vol. 48, pp. 497-513, 2003.

[34] D. Caudwell, J. Trusler, V. Vesovic, and W. Wakeham, "The viscosity and density of n-Dodecane and n-Octadecane at pressures up to $200 \mathrm{MPa}$ and temperatures up to 473K," Int. J. Thermophys., vol. 25, pp. 1339-1352, 2004.

[35] W. Seyer, R. Patterson, and J. Keays, "The density and transition points of the n-paraffin hydrocarbons," J. Am. Chem. Soc., vol. 66, pp. 179-182, 1944.

[36] C. Yaws, Transport Properties of Chemicals and Hydrocarbons, ch. 3. William Andrew, 2009.

[37] J. Marano and G. Holder, "A general equation for correlating the thermophysical properties of nparaffins, n-olefins, and other homologous series. 3. Asymptotic behavior correlations for thermal and transport properties.," Ind. Eng. Chem. Res., vol. 36, pp. 2399-2408, 1997.

[38] R. Holmen, M. Lamvik, and O. Melhus, "Measurements of the thermal conductivities of solid and liquid unbranched alkanes in the C16-to-C19 range during phase transition," Int. J. Thermophys., vol. 23, pp. 27-39, 2002. 
[39] A. Briard, M. Bouroukba, Petitjean, and M. Dirand, "Models for estimation of pure n-alkanes' thermodynamic properties as a function of carbon chain length," J. Chem. Eng. Data, vol. 48, pp. 1508-1516, 2003.

[40] D. Ducoulombier, H. Zhou, C. Boned, J. Peyrelasse, H. Saint-Guirons, and P. Xans, "Pressure (1-1000 bars) and temperature $\left(20-100^{\circ} \mathrm{C}\right)$ dependence of the viscosity of liquid hydrocarbons," J. Phys. Chem., vol. 90, pp. 1692-1700, 1986.

[41] H. Forsman and P. Andersson, "Thermal conductivity at high pressure of solid odd-numbered n-alkanes ranging from C9H20 to C19H40," J. Chem. Phys., vol. 80, pp. 2804-2807, 1984. 